TRANSACTIONS OF THE

AMERICAN MATHEMATICAL SOCIETY

Volume 357, Number 10, Pages 4191-4213

S 0002-9947(04)03666-9

Article electronically published on October 28, 2004

\title{
MINKOWSKI VALUATIONS
}

\author{
MONIKA LUDWIG
}

\begin{abstract}
Centroid and difference bodies define SL $(n)$ equivariant operators on convex bodies and these operators are valuations with respect to Minkowski addition. We derive a classification of $\mathrm{SL}(n)$ equivariant Minkowski valuations and give a characterization of these operators. We also derive a classification of SL $(n)$ contravariant Minkowski valuations and of $L_{p}$-Minkowski valuations.
\end{abstract}

Centroid, difference, and projection bodies are fundamental notions in the affine geometry of convex bodies. The most important affine isoperimetric inequalities (and open problems) are formulated using these bodies. We show that the operators defined by these bodies together with the identity are basically the only examples of homogeneous, $\mathrm{SL}(n)$ equivariant or contravariant Minkowski valuations.

The centroid body $\Gamma K$ of a convex body $K \subset \mathbb{R}^{n}$ is a classical notion from geometry (see [5], [16], 36]) that has attracted much attention in recent years (see [4], [6], 8], [20], 21], 25], 27], 31]). If $K$ is $o$-symmetric, then $\Gamma K$ is the body whose boundary consists of the locus of the centroids of the halves of $K$ formed when $K$ is cut by hyperplanes through the origin. In general it can be defined in the following way. Let $\mathcal{K}^{n}$ denote the set of convex bodies (that is, of compact, convex sets) in $\mathbb{R}^{n}$, and let $\mathcal{K}_{o}^{n}$ denote the set of convex bodies in $\mathbb{R}^{n}$ that contain the origin. A convex body $K$ is uniquely determined by its support function $h(K, \cdot)$, where $h(K, v)=\max \{v \cdot x: x \in K\}, v \in \mathbb{R}^{n}$, and where $v \cdot x$ denotes the standard inner product of $v$ and $x$. The moment body $\mathrm{M} K$ of $K \in \mathcal{K}_{o}^{n}$ is the convex body whose support function is given by

$$
h(\mathrm{M} K, v)=\int_{K}|v \cdot x| d x .
$$

If the $n$-dimensional volume $\operatorname{vol}_{n}(K)$ of $K$ is positive, then the centroid body $\Gamma K$ of $K$ is defined by

$$
\Gamma K=\frac{1}{\operatorname{vol}_{n}(K)} \mathrm{M} K .
$$

The fundamental affine isoperimetric inequality for centroid bodies is the Busemann-Petty centroid inequality [32]: Among bodies of given volume precisely for centered ellipsoids the centroid bodies have minimal volume. It is one of the major open problems to determine the reverse inequality (see [31]).

The difference body $\mathrm{D} K$ of $K \in \mathcal{K}^{n}$ is the Minkowski (or vector) sum of $K$ and its reflection in the origin, that is,

$$
\mathrm{D} K=K+(-K)
$$

Received by the editors December 17, 2003.

2000 Mathematics Subject Classification. Primary 52A20; Secondary 52B11, 52B45.

(C)2004 American Mathematical Society 
The operation that forms the difference body is essentially that known as central symmetrization and as such finds many applications in geometry and mathematical physics. The fundamental affine isoperimetric inequality for difference bodies is the Rogers-Shephard inequality [34]: Among bodies of given volume precisely for simplices the difference bodies have maximal volume.

The moment and difference operators are both Minkowski valuations. Here an operator $\mathrm{Z}$ is called a Minkowski valuation if

$$
\mathrm{Z} K_{1}+\mathrm{Z} K_{2}=\mathrm{Z}\left(K_{1} \cup K_{2}\right)+\mathrm{Z}\left(K_{1} \cap K_{2}\right),
$$

whenever $K_{1}, K_{2}, K_{1} \cup K_{2} \in \mathcal{K}^{n}$ and addition on $\mathcal{K}^{n}$ is Minkowski addition. Valuations on convex bodies are a classical concept. In 1900, Dehn used them for solving Hilbert's third problem on the non-equidecomposability of convex polytopes of equal volume in $\mathbb{R}^{3}$. Probably the most famous result on valuations is Hadwiger's characterization of rigid motion invariant real valued valuations continuous with respect to the Hausdorff metric as linear combinations of quermassintegrals. See 9], 15, 29], 30 for information on the classical theory and [1-3], 11- 14], [18, [19], 37] for some of the more recent results.

An operator $\mathrm{Z}: \mathcal{K}^{n} \rightarrow \mathcal{K}^{n}$ is Minkowski additive if $\mathrm{Z}\left(K_{1}+K_{2}\right)=\mathrm{Z} K_{1}+\mathrm{Z} K_{2}$ for $K_{1}, K_{2} \in \mathcal{K}^{n}$. Note that every Minkowski additive operator is a Minkowski valuation but not vice versa. Continuous Minkowski additive operators that commute with rigid motions are called endomorphisms. Schneider [35] (see also [36]) showed that there is a great variety of these operators. He obtained a complete classification of endomorphisms in $\mathcal{K}^{2}$ and characterizations of special endomorphisms in $\mathcal{K}^{n}$. These results were further extended by Kiderlen [10].

We show that the moment and difference operators are basically the only examples of homogeneous, $\mathrm{SL}(n)$ equivariant Minkowski valuations. Here an operator $\mathrm{Z}: \mathcal{K}_{o}^{n} \rightarrow \mathcal{K}^{n}$ is called SL $(n)$ equivariant, if

$$
\mathrm{Z}(\phi K)=\phi \mathrm{Z} K \quad \text { for } \phi \in \mathrm{SL}(n),
$$

and it is called homogeneous of degree $r, r \in \mathbb{R}$, if

$$
\mathrm{Z}(s K)=s^{r} \mathrm{Z} K \quad \text { for } s \geq 0 \text {. }
$$

Let $\mathcal{P}_{o}^{n}$ denote the set of convex polytopes in $\mathbb{R}^{n}$ that contain the origin. For $n \geq 3$, we show that $\mathrm{Z}: \mathcal{P}_{o}^{n} \rightarrow \mathcal{K}^{n}$ is a homogeneous, $\mathrm{SL}(n)$ equivariant Minkowski valuation if and only if there are constants $c_{0} \in \mathbb{R}, c_{1}, c_{2} \geq 0$ such that

$$
\mathrm{Z} P=c_{0} m(P)+c_{1} \mathrm{M} P \text { or } \quad \mathrm{Z} P=c_{1} P+c_{2}(-P)
$$

for every $P \in \mathcal{P}_{o}^{n}$, where $m(P)$ is the moment vector of $P$. In particular, this implies that these are all continuous, homogeneous, $\operatorname{SL}(n)$ equivariant Minkowski valuations on $\mathcal{K}_{o}^{n}$. Combined with McMullen's polynomial expansion for translation invariant valuations 28 , it implies that $\mathrm{Z}: \mathcal{P}^{n} \rightarrow \mathcal{K}^{n}$ is a translation invariant, $\mathrm{SL}(n)$ equivariant Minkowski valuation if and only if there is a constant $c \geq 0$ such that

$$
\mathrm{Z} P=c \mathrm{D} P
$$

for every $P \in \mathcal{P}^{n}$. We also derive the corresponding results in the context of the $L_{p}$-Brunn-Minkowski theory and give a characterization of $L_{p}$-centroid bodies.

The projection body $\Pi K$ of $K$ is the convex body whose support function is given for $u \in S^{n-1}$ by

$$
h(\Pi K, u)=\operatorname{vol}_{n-1}\left(K \mid u^{\perp}\right),
$$


where $\operatorname{vol}_{n-1}$ denotes $(n-1)$-dimensional volume and $K \mid u^{\perp}$ denotes the image of the orthogonal projection of $K$ onto the subspace orthogonal to $u$. Projection bodies, which were introduced by Minkowski, are an important tool for studying projections (see [5]). In recent years, projection bodies and their generalizations in the $L_{p}$-Brunn-Minkowski theory have attracted increased attention; see [7], [25], [26], [39]. The fundamental affine isoperimetric inequalities for projection bodies are the Petty projection inequality [33] and the Zhang projection inequality [38]: Among bodies of given volume precisely for ellipsoids the polar projection bodies have maximal volume and precisely for simplices the polar projection bodies have minimal volume. It is a major open problem to determine the corresponding results for the volume of the projection body itself (see [23]).

We derive a classification of homogeneous, $\mathrm{SL}(n)$ contravariant Minkowski valuations and give a characterization of the projection operator. Here an operator $\mathrm{Z}: \mathcal{K}_{o}^{n} \rightarrow \mathcal{K}^{n}$ is called SL $(n)$ contravariant, if

$$
\mathrm{Z}(\phi K)=\phi^{-t} \mathrm{Z} K \quad \text { for } \phi \in \mathrm{SL}(n),
$$

where $\phi^{-t}$ denotes the inverse of the transpose of $\phi$. This classification generalizes a result in [17] where it is shown that an operator $\mathrm{Z}: \mathcal{P}^{n} \rightarrow \mathcal{P}^{n}$ is a Minkowski valuation that is $\mathrm{SL}(n)$ contravariant, translation invariant and homogeneous of degree $(n-1)$ if and only if it is a multiple of the projection operator. Here we obtain that an operator $\mathrm{Z}: \mathcal{P}^{n} \rightarrow \mathcal{K}^{n}$ is a translation invariant, $\mathrm{SL}(n)$ contravariant Minkowski valuation if and only if there is a constant $c \geq 0$ such that

$$
\mathrm{Z} P=c \Pi P
$$

for every $P \in \mathcal{P}^{n}$. We also derive the corresponding results in the context of the $L_{p}$-Brunn-Minkowski theory.

\section{Equivariant Minkowski valuations}

In this section, our main result on the classification of SL $(n)$ equivariant Minkowski valuations is formulated. The important examples are the difference operator, the moment operator and the moment vector. Here the moment vector $m(K)$ of a convex body $K \in \mathcal{K}_{o}^{n}$ is defined by

$$
m(K)=\int_{K} x d x .
$$

Note that the moment operator $\mathrm{M}: \mathcal{K}_{o}^{n} \rightarrow \mathcal{K}^{n}$ and the moment vector $m: \mathcal{K}_{o}^{n} \rightarrow \mathbb{R}^{n}$ commute (up to a determinantal factor) with general linear transformations:

$$
\mathrm{M}(\phi K)=|\operatorname{det} \phi| \phi \mathrm{M} K \text { and } m(\phi K)=|\operatorname{det} \phi| \phi m(K) \quad \text { for } \phi \in \mathrm{GL}(n) .
$$

The difference operator and the identity are $\mathrm{SL}(n)$ equivariant and homogeneous of degree 1 . As we will see, for $n \geq 3$ these are already all examples of homogeneous, $\mathrm{SL}(n)$ equivariant Minkowski valuations on $\mathcal{P}_{o}^{n}$.

For $n=2$, we have an additional example. Let $\mathcal{E}_{o}(P)$ be the set of edges of $P$ that contain the origin. For $a_{0}, b_{0} \geq 0$ and $a_{i}, b_{i} \in \mathbb{R}$ with $a_{i}+b_{0}+b_{1}, a_{0}+a_{1}+b_{i} \geq 0$, $i=1,2$, define $\mathrm{Z}: \mathcal{P}_{o}^{2} \rightarrow \mathcal{P}_{o}^{2}$ by

$$
\mathrm{Z} P=a_{0} P+b_{0}(-P)+\sum\left(a_{i} E_{i}+b_{i}\left(-E_{i}\right)\right),
$$


where the sum is taken over $E_{i} \in \mathcal{E}_{o}(P)$. Here and throughout formulae like (11) have to be read as

$$
h(\mathrm{Z} P, v)=a_{0} h(P, v)+b_{0} h(-P, v)+\sum\left(a_{i} h\left(E_{i}, v\right)+b_{i} h\left(-E_{i}, v\right)\right)
$$

for $v \in \mathbb{R}^{2}$. The notation is only used if $h(\mathrm{Z} P, \cdot)$ is the support function of a convex body, which is here guaranteed by the conditions on $a_{i}, b_{i}$. Note that $\mathrm{Z}: \mathcal{P}_{o}^{2} \rightarrow$ $\mathcal{K}^{2}$ defined by (1) is $\mathrm{SL}(2)$ equivariant, homogeneous of degree 1 , and Minkowski additive.

The following result is our classification of $\mathrm{SL}(n)$ equivariant Minkowski valuations. The proof is given in Section 3

Theorem 1. Let $\mathrm{Z}: \mathcal{P}_{o}^{n} \rightarrow \mathcal{K}^{n}, n \geq 3$, be a Minkowski valuation which is $\mathrm{SL}(n)$ equivariant and homogeneous of degree $r$. If $r=n+1$, then there are constants $a_{0} \in \mathbb{R}, a_{1} \geq 0$ such that

$$
\mathrm{Z} P=a_{0} m(P)+a_{1} \mathrm{M} P
$$

for every $P \in \mathcal{P}_{o}^{n}$. If $r=1$, then there are constants $a, b \geq 0$ such that

$$
\mathrm{Z} P=a P+b(-P)
$$

for every $P \in \mathcal{P}_{o}^{n}$. In all other cases, $\mathrm{Z} P=\left\{\right.$ o for every $P \in \mathcal{P}_{o}^{n}$.

Let $\mathrm{Z}: \mathcal{P}_{o}^{2} \rightarrow \mathcal{K}^{2}$ be a Minkowski valuation which is $\mathrm{SL}(2)$ equivariant and homogeneous of degree $r$. If $r=3$, then there are constants $a_{0} \in \mathbb{R}, a_{1} \geq 0$ such that

$$
\mathrm{Z} P=a_{0} m(P)+a_{1} \mathrm{M} P
$$

for every $P \in \mathcal{P}_{o}^{2}$. If $r=1$, then there are constants $a_{0}, b_{0} \geq 0$ and $a_{i}, b_{i} \in \mathbb{R}$ with $a_{i}+b_{0}+b_{1}, a_{0}+a_{1}+b_{i} \geq 0, i=1,2$, such that

$$
\mathrm{Z} P=a_{0} P+b_{0}(-P)+\sum\left(a_{i} E_{i}+b_{i}\left(-E_{i}\right)\right)
$$

for every $P \in \mathcal{P}_{o}^{2}$, where the sum is taken over $E_{i} \in \mathcal{E}_{o}(P)$. In all other cases, $\mathrm{Z} P=\{$ o $\}$ for every $P \in \mathcal{P}_{o}^{2}$.

Let $\mathcal{K}^{n}$ and $\mathcal{K}_{o}^{n}$ be equipped with the topology defined by the Hausdorff metric. We have the following simple consequence of Theorem 1. Note that there are further examples of homogeneous, $\mathrm{SL}(n)$ equivariant Minkowski valuations on $\mathcal{K}_{o}^{n}$ that are not continuous (see [35]).

Corollary 1.1. If $\mathrm{Z}: \mathcal{K}_{o}^{n} \rightarrow \mathcal{K}^{n}, n \geq 2$, is a continuous, homogeneous, $\operatorname{SL}(n)$ equivariant Minkowski valuation, then there are constants $a_{0} \in \mathbb{R}, a_{1}, a_{2} \geq 0$ such that

$$
\mathrm{Z} K=a_{0} m(K)+a_{1} \mathrm{M} K \quad \text { or } \quad \mathrm{Z} K=a_{1} K+a_{2}(-K)
$$

for every $K \in \mathcal{K}_{o}^{n}$.

For translation invariant valuations, we obtain the following result.

Corollary 1.2. If $\mathrm{Z}: \mathcal{P}^{n} \rightarrow \mathcal{K}^{n}, n \geq 2$, is a translation invariant, $\mathrm{SL}(n)$ equivariant Minkowski valuation, then there is a constant $c \geq 0$ such that $\mathrm{Z}=c \mathrm{D}$.

Here an operator $\mathrm{Z}: \mathcal{K}^{n} \rightarrow \mathcal{K}^{n}$ is called translation invariant, if $\mathrm{Z}(K+x)=\mathrm{Z} K$ for $x \in \mathbb{R}^{n}$. The proof of this corollary is given in Section 7 


\section{Equivariant $L_{p}$-Minkowski VALUATions}

For $p>1$, the $L_{p}$-Minkowski sum $K_{1}+{ }_{p} K_{2}$ of $K_{1}, K_{2} \in \mathcal{K}_{o}^{n}$ is defined by

$$
h^{p}\left(K_{1}+{ }_{p} K_{2}, v\right)=h^{p}\left(K_{1}, v\right)+h^{p}\left(K_{2}, v\right)
$$

for $v \in \mathbb{R}^{n}$. This notion, which was introduced by Firey in the middle of the last century, is at the heart of the $L_{p}$-Brunn-Minkowski theory (or Brunn-MinkowskiFirey theory); see [22], 24]. We derive a classification of homogeneous, $\operatorname{SL}(n)$ equivariant $L_{p}$-Minkowski valuations. Here an operator $\mathrm{Z}: \mathcal{K}_{o}^{n} \rightarrow \mathcal{K}_{o}^{n}$ is called an $L_{p}$-Minkowski valuation if

$$
\mathrm{Z} K_{1}+{ }_{p} \mathrm{Z} K_{2}=\mathrm{Z}\left(K_{1} \cup K_{2}\right)+_{p} \mathrm{Z}\left(K_{1} \cap K_{2}\right),
$$

whenever $K_{1}, K_{2}, K_{1} \cup K_{2} \in \mathcal{K}_{o}^{n}$.

The important examples of $\mathrm{SL}(n)$ equivariant $L_{p}$-Minkowski valuations are the $L_{p}$-moment operator and the $L_{p}$-difference operator. These can be defined in the following way. For $-1 \leq \tau \leq 1$, define $\mathrm{M}_{p}^{\tau}: \mathcal{K}_{o}^{n} \rightarrow \mathcal{K}_{o}^{n}$ by

$$
h^{p}\left(\mathrm{M}_{p}^{\tau} K, v\right)=\int_{K}(|v \cdot x|+\tau(v \cdot x))^{p} d x
$$

for $v \in \mathbb{R}^{n}$. For $\tau=0$, we obtain the $L_{p}$-moment operator $\mathrm{M}_{p}$. If $\operatorname{vol}_{n}(K)>0$, then the $L_{p}$-centroid body $\Gamma_{p} K$ of $K$ is defined by

$$
\Gamma_{p} K=\frac{c_{n, p}}{\operatorname{vol}_{n}(K)} \mathrm{M}_{p} K,
$$

where the constant $c_{n, p}$ is chosen such that $\Gamma_{p} B=B$ for the unit ball $B . L_{p^{-}}$ centroid bodies were introduced by Lutwak and Zhang [27], for $p=2$ they are the Legendre ellipsoids of classical mechanics. Lutwak, Yang, and Zhang [25] obtained the $L_{p}$-version of the Busemann-Petty centroid inequality; see Campi and Gronchi 4] for a different proof. Note that

$$
\mathrm{M}_{p}^{\tau}(\phi K)=|\operatorname{det} \phi|^{1 / p} \phi \mathrm{M}_{p}^{\tau} K \quad \text { for } \phi \in \mathrm{GL}(n),
$$

and that $\mathrm{M}_{p}^{\tau}$ is an $L_{p}$-Minkowski valuation. The $L_{p}$-difference operator $\mathrm{D}_{p}$, defined by

$$
\mathrm{D}_{p} K=K+_{p}(-K)
$$

for $K \in \mathcal{K}_{o}^{n}$, and the identity are $\operatorname{SL}(n)$ equivariant, homogeneous of degree 1 and $L_{p}$-Minkowski valuations. We will show that for $n \geq 3$ these are all examples.

For $n=2$, there are additional examples. For $a_{0}, b_{0} \geq 0$ and $a_{i}, b_{i} \in \mathbb{R}$ with $a_{0}+a_{i}, b_{0}+b_{i} \geq 0, i=1,2$, define $\mathrm{Z}: \mathcal{P}_{o}^{2} \rightarrow \mathcal{K}_{o}^{2}$ by

$$
\mathrm{Z} P=a_{0} P+_{p} b_{0}(-P)+_{p} \sum^{p}\left(a_{i} E_{i}+_{p} b_{i}\left(-E_{i}\right)\right)
$$

for every $P \in \mathcal{P}_{o}^{2}$, where the sum is taken over $E_{i} \in \mathcal{E}_{o}(P)$. Here $\sum^{p}$ denotes the $L_{p}$-Minkowski sum. Then $\mathrm{Z}: \mathcal{P}_{o}^{2} \rightarrow \mathcal{K}_{o}^{2}$ is $\mathrm{SL}(2)$ equivariant, homogeneous of degree 1 , and $L_{p}$-Minkowski additive. A further operator is obtained in the following way. Let $\psi_{\pi / 2}$ denote the rotation by an angle $\pi / 2$ and for $-1 \leq \tau \leq 1$, let $\hat{\Pi}_{p}^{\tau}$ be the operator defined in Section 5 Since $\hat{\Pi}_{p}^{\tau}$ is $\operatorname{SL}(2)$ contravariant, we have

$$
\left(\psi_{\pi / 2} \hat{\Pi}_{p}^{\tau}\right)(\phi P)=\psi_{\pi / 2} \phi^{-t} \psi_{\pi / 2}^{-1}\left(\psi_{\pi / 2} \hat{\Pi}_{p}^{\tau}\right) P=\phi\left(\psi_{\pi / 2} \hat{\Pi}_{p}^{\tau}\right) P
$$

that is, $\psi_{\pi / 2} \hat{\Pi}_{p}^{\tau}$ is $\operatorname{SL}(2)$ equivariant. Since $\hat{\Pi}_{p}^{\tau}$ is homogeneous of degree $2 / p-1$, an $L_{p}$-Minkowski valuation and not continuous, so is $\psi_{\pi / 2} \hat{\Pi}_{p}^{\tau}$. 
The following result is our classification of $\mathrm{SL}(n)$ equivariant $L_{p}$-Minkowski valuations. The proof is given in Section 3

Theorem $\mathbf{1}_{\mathbf{p}}$. Let $\mathrm{Z}: \mathcal{P}_{o}^{n} \rightarrow \mathcal{K}_{o}^{n}, n \geq 3$, be an $L_{p}$-Minkowski valuation, $p>1$, which is $\mathrm{SL}(n)$ equivariant and homogeneous of degree $r$. If $r=n / p+1$, then there are constants $a \geq 0$ and $-1 \leq \tau \leq 1$ such that

$$
\mathrm{Z} P=a \mathrm{M}_{p}^{\tau} P
$$

for every $P \in \mathcal{P}_{o}^{n}$. If $r=1$, then there are constants $a, b \geq 0$ such that

$$
\mathrm{Z} P=a P+{ }_{p} b(-P)
$$

for every $P \in \mathcal{P}_{o}^{n}$. In all other cases, $\mathrm{Z} P=\{o\}$ for every $P \in \mathcal{P}_{o}^{n}$.

Let $\mathrm{Z}: \mathcal{P}_{o}^{2} \rightarrow \mathcal{K}_{o}^{2}$ be an $L_{p}$-Minkowski valuation, $p>1$, which is $\mathrm{SL}(2)$ equivariant and homogeneous of degree $r$. If $r=2 / p+1$, then there are constants $a \geq 0$ and $-1 \leq \tau \leq 1$ such that

$$
\mathrm{Z} P=a \mathrm{M}_{p}^{\tau} P
$$

for every $P \in \mathcal{P}_{o}^{2}$. If $r=1$, then there are constants $a_{0}, b_{0} \geq 0$ and $a_{i}, b_{i} \in \mathbb{R}$ with $a_{0}+a_{i}, b_{0}+b_{i} \geq 0, i=1,2$, such that

$$
\mathrm{Z} P=a_{0} P+_{p} b_{0}(-P)+{ }_{p} \sum^{p}\left(a_{i} E_{i}+_{p} b_{i}\left(-E_{i}\right)\right)
$$

for every $P \in \mathcal{P}_{o}^{2}$, where the sum is taken over $E_{i} \in \mathcal{E}_{o}(P)$. If $r=2 / p-1$, then there are constants $a \geq 0$ and $-1 \leq \tau \leq 1$ such that

$$
\mathrm{Z} P=a \psi_{\pi / 2} \hat{\Pi}_{p}^{\tau} P
$$

for every $P \in \mathcal{P}_{o}^{2}$. In all other cases, $\mathrm{Z} P=\{$ o $\}$ for every $P \in \mathcal{P}_{o}^{2}$.

As a simple consequence we obtain the following corollary.

Corollary. If $\mathrm{Z}: \mathcal{K}_{o}^{n} \rightarrow \mathcal{K}_{o}^{n}, n \geq 2$, is a continuous, homogeneous, $\mathrm{SL}(n)$ equivariant $L_{p}$-Minkowski valuation, $p>1$, then there are constants $a, b \geq 0$ and $-1 \leq \tau \leq 1$ such that

$$
\mathrm{Z} K=a \mathrm{M}_{p}^{\tau} K \quad \text { or } \quad \mathrm{Z} K=a K+_{p} b(-K)
$$

for every $K \in \mathcal{K}_{o}^{n}$.

\section{Proof of Theorems 1 and 1 p}

In the following, we work in $n$-dimensional Euclidean space $\mathbb{R}^{n}$ with origin $o$, a fixed orthonormal basis $e_{1}, \ldots, e_{n}$, and use coordinates $x=\left(x_{1}, \ldots, x_{n}\right)$ for $x \in \mathbb{R}^{n}$. An operator $\mathrm{Z}: \mathcal{P}_{o}^{n} \rightarrow \mathcal{K}^{n}$ is called simple, if $\mathrm{Z} P=\{o\}$ for every $P \in \mathcal{P}_{o}^{n}$ with $\operatorname{dim} P<n$. Here $\operatorname{dim} P$ is the dimension of the linear hull, $\operatorname{lin} P$, of $P$. As a first step in the proof, we show that every operator which is $\mathrm{SL}(n)$ equivariant and homogeneous of degree $r \neq 1$ is simple.

Lemma 1. Let $\mathrm{Z}: \mathcal{P}_{o}^{n} \rightarrow \mathcal{K}^{n}$ be an operator which is $\mathrm{SL}(n)$ equivariant and homogeneous of degree $r$. Then $\mathrm{Z} P \subset \operatorname{lin} P$. If $\operatorname{dim} P \leq(n-1)$ and $r \neq 1$, then $\mathrm{Z} P=\{o\}$.

Proof. Let $P \in \mathcal{P}_{o}^{n}$ be such that $\operatorname{lin} P$ is the $k$-dimensional subspace with equation $x_{k+1}=\ldots=x_{n}=0$. Since every $P^{\prime} \in \mathcal{P}_{o}^{n}$ with $\operatorname{dim} P^{\prime}=k$ is a linear image of 
such a polytope $P$ and since $\mathrm{Z}$ is $\mathrm{SL}(n)$ equivariant, it suffices to prove the lemma in this case. Let

$$
\phi=\left(\begin{array}{cc}
I & B \\
0 & A
\end{array}\right),
$$

where $I$ is the $k \times k$ identity matrix, 0 is the $(n-k) \times k$ zero matrix, $B$ is an $(n-k) \times k$ matrix, and $A$ is an $(n-k) \times(n-k)$ matrix with determinant 1 . Then $\phi \in \mathrm{SL}(n)$ and

$$
\phi P=P .
$$

Write $x=\left(x^{\prime}, x^{\prime \prime}\right)$ with $x^{\prime}=\left(x_{1}, \ldots, x_{k}\right)$ and $x^{\prime \prime}=\left(x_{k+1}, \ldots, x_{n}\right)$ for $x \in \mathbb{R}^{n}$. Let $x \in \mathrm{Z} P$. Since Z is $\mathrm{SL}(n)$ equivariant, (2) implies that $y=\phi x \in \mathrm{Z} P$. Therefore

$$
\left(\begin{array}{c}
y^{\prime} \\
y^{\prime \prime}
\end{array}\right)=\left(\begin{array}{c}
x^{\prime}+B x^{\prime \prime} \\
A x^{\prime \prime}
\end{array}\right) \in \mathrm{ZP} .
$$

This is true for every $(n-k) \times k$ matrix $B$ and every $(n-k) \times(n-k)$ matrix $A$ with determinant 1 . If $x^{\prime \prime} \neq o^{\prime \prime}$, this implies that $y^{\prime}$ can be an arbitrary vector. Since Z $P$ is bounded, it follows that $x^{\prime \prime}=o^{\prime \prime}$. Thus Z $P \subset \operatorname{lin} P$.

Now, let $r \neq 1$ and let

$$
\phi=\left(\begin{array}{ll}
I & 0 \\
0 & s
\end{array}\right),
$$

where $I$ is the $(n-1) \times(n-1)$ identity matrix and $s \geq 0$. Then

$$
\mathrm{Z}(\phi P)=s^{(r-1) / n} \mathrm{Z} P=\mathrm{Z} P .
$$

Since this holds for every $s \geq 0$ and $\mathrm{Z} P$ is bounded, this implies that $\mathrm{Z} P=\{o\}$.

Next, we show that we can reduce the proof of Theorem 1 to showing that the corresponding results hold for simplices. The arguments used in the proof of the next lemma are well known from several extension theorems for valuations (cf. [9] or [15]).

Lemma 2. Let $\mathrm{Z}_{1}, \mathrm{Z}_{2}: \mathcal{P}_{o}^{n} \rightarrow \mathcal{K}^{n}$ be Minkowski valuations. If $\mathrm{Z}_{1} S=\mathrm{Z}_{2} S$ for every $n$-dimensional simplex $S \in \mathcal{P}_{o}^{n}$, then $\mathrm{Z}_{1}=\mathrm{Z}_{2}$.

Proof. If $T^{\prime} \in \mathcal{P}_{o}^{n}$ is a simplex with $\operatorname{dim} T^{\prime}=k<n$, then there is a simplex $T$ such that $T^{\prime}=T \cap H$, where $H=\operatorname{lin} T^{\prime}$ and such that $T \cap H^{+}$and $T \cap H^{-}$are both $(k+1)$-dimensional. Here $H^{+}, H^{-}$denote the closed halfspaces bounded by $H$. Since $\mathrm{Z}_{i}$ is a valuation, we have for $i=1,2$

$$
\mathrm{Z}_{i} T+\mathrm{Z}_{i} T^{\prime}=\mathrm{Z}_{i}\left(T \cap H^{+}\right)+\mathrm{Z}_{i}\left(T \cap H^{-}\right) .
$$

If $\mathrm{Z}_{1} S=\mathrm{Z}_{2} S$ for every $(k+1)$-dimensional simplex $S$, this implies that $\mathrm{Z}_{1} T^{\prime}=$ $\mathrm{Z}_{2} T^{\prime}$. Thus we get by induction that $\mathrm{Z}_{1} T=\mathrm{Z}_{2} T$ for every simplex $T \in \mathcal{P}_{o}^{n}$.

For $x \in \mathbb{R}^{n}$ and $i, i=1,2$, fixed, $\mu(P)=h\left(\mathrm{Z}_{i} P, x\right)$ is a real valued valuation. Let $P \in \mathcal{P}_{o}^{n}$ be dissected into $n$-dimensional polytopes $P_{1}, \ldots P_{m} \in \mathcal{P}_{o}^{n}$, that is, $P=P_{1} \cup \ldots \cup P_{m}$ and the $P_{i}$ 's have pairwise disjoint interiors. Induction on the dimension and the number of terms shows that the inclusion-exclusion principle

$$
\mu(P)=\sum_{I}(-1)^{|I|-1} \mu\left(P_{I}\right)
$$

holds, where the sum is taken over all ordered $k$-tuples $I=\left\{i_{1}, \ldots, i_{k}\right\}$ such that $1 \leq i_{1}<\ldots<i_{k} \leq n$ and $k=1, \ldots, m$. Here $|I|$ denotes the cardinality of $I$ and $P_{I}=P_{i_{1}} \cap \ldots \cap P_{i_{k}}$ (cf. [15]). 
Let $P \in \mathcal{P}_{o}^{n}$. If $\operatorname{dim} P=n$, then we dissect $P$ into $n$-dimensional simplices $S_{i} \in \mathcal{P}_{n}, i=1, \ldots, m$. Then (4) implies that $\mathrm{Z}_{1} P=\mathrm{Z}_{2} P$. If $\operatorname{dim} P<k$, we use the same $\operatorname{argument}$ in $\operatorname{lin} P$.

The following lemma is used to prove Theorem 1, The proof is same as that of Lemma 2

Lemma $\mathbf{2}_{\mathbf{p}}$. Let $\mathrm{Z}_{1}, \mathrm{Z}_{2}: \mathcal{P}_{o}^{n} \rightarrow \mathcal{K}_{o}^{n}$ be $L_{p}$-Minkowski valuations. If $\mathrm{Z}_{1} S=\mathrm{Z}_{2} S$ for every $n$-dimensional simplex $S \in \mathcal{P}_{o}^{n}$, then $\mathrm{Z}_{1}=\mathrm{Z}_{2}$.

To prove Theorems 1 and $\mathbb{1}_{\mathrm{p}}$ we determine the value of $\mathrm{Z}$ for $n$-dimensional simplices. Since $\mathrm{Z}$ is $\mathrm{SL}(n)$ equivariant and homogeneous, it is enough to consider a standard simplex. So let $T$ be the simplex with vertices $o, e_{1}, \ldots, e_{n}$.

We start with the planar case.

Proposition 1. Let $\mathrm{Z}: \mathcal{P}_{o}^{2} \rightarrow \mathcal{K}^{2}$ be a Minkowski valuation which is $\mathrm{SL}(2)$ equivariant and homogeneous of degree $r$. If $r=3$, then there are constants $a_{0} \in \mathbb{R}$, $a_{1} \geq 0$ such that

$$
\mathrm{Z} T=a_{0} m(T)+a_{1} \mathrm{M} T .
$$

If $r=1$, then there are constants $a_{0}, b_{0} \geq 0$ and $a_{i}, b_{i} \in \mathbb{R}$ with $a_{i}+b_{0}+b_{1}, a_{0}+$ $a_{2}+b_{i} \geq 0, i=1,2$, such that

$$
\mathrm{ZT}=a_{0} T+b_{0}(-T)+a_{1}\left[o, e_{1}\right]+b_{1}\left[o,-e_{1}\right]+a_{2}\left[o, e_{2}\right]+b_{2}\left[o,-e_{2}\right] .
$$

In all other cases, $\mathrm{ZT}=\{o\}$.

Proposition $\mathbf{1}_{\mathbf{p}}$. Let $\mathrm{Z}: \mathcal{P}_{o}^{2} \rightarrow \mathcal{K}_{o}^{2}$ be an $L_{p}$-Minkowski valuation, $p>1$, which is $\mathrm{SL}(2)$ equivariant and homogeneous of degree $r$. If $r=2 / p+1$, then there are constants $a \geq 0$ and $-1 \leq \tau \leq 1$ such that

$$
\mathrm{Z} T=a \mathrm{M}_{p}^{\tau} T .
$$

If $r=1$, then there are constants $a_{0}, b_{0} \geq 0$ and $a_{i}, b_{i} \in \mathbb{R}$ with $a_{0}+a_{i}, b_{0}+b_{i} \geq 0$, $i=1,2$, such that

$$
\mathrm{ZT}=a_{0} T+{ }_{p} b_{0}(-T)+{ }_{p} a_{1}\left[o, e_{1}\right]+{ }_{p} b_{1}\left[o,-e_{1}\right]+{ }_{p} a_{2}\left[o, e_{2}\right]+{ }_{p} b_{2}\left[o,-e_{2}\right] .
$$

If $r=2 / p-1$, then there are constants $a_{1}, a_{2} \geq 0$ such that

$$
\mathrm{ZT}=\left[-a_{1}\left(e_{1}-e_{2}\right), a_{2}\left(e_{1}-e_{2}\right)\right] .
$$

In all other cases, $\mathrm{Z} T=\{o\}$.

Proposition 1 is basically a special case of Proposition 1. The only difference is the range of the operators. We prove both propositions at the same time.

Proof. If $p>1$, let $\mathrm{Z}: \mathcal{P}_{o}^{2} \rightarrow \mathcal{K}_{o}^{2}$, and if $p=1$, let $\mathrm{Z}: \mathcal{P}_{o}^{2} \rightarrow \mathcal{K}^{2}$.

For $0<\lambda<1$, let $H_{\lambda}$ be the hyperplane through the origin $o$ with normal vector $(1-\lambda) e_{1}-\lambda e_{2}$. Then $H_{\lambda}$ dissects $T$ into two simplices $T \cap H_{\lambda}^{+}$and $T \cap H_{\lambda}^{-}$, where $H_{\lambda}^{+}, H_{\lambda}^{-}$are the closed halfspaces bounded by $H_{\lambda}$. Since $\mathrm{Z}$ is a valuation, we have

$$
\mathrm{Z} T+{ }_{p} \mathrm{Z}\left(T \cap H_{\lambda}\right)=\mathrm{Z}\left(T \cap H_{\lambda}^{+}\right)+{ }_{p} \mathrm{Z}\left(T \cap H_{\lambda}^{-}\right) .
$$

Set $T^{\prime}=T \cap e_{1}^{\perp}$, where $e_{1}^{\perp}$ denotes the subspace orthogonal to $e_{1}$, and set

$$
\phi_{\lambda}=\left(\begin{array}{cc}
\lambda & 0 \\
1-\lambda & 1
\end{array}\right) \quad \text { and } \quad \psi_{\lambda}=\left(\begin{array}{cc}
1 & \lambda \\
0 & 1-\lambda
\end{array}\right) .
$$


Then $T \cap H_{\lambda}=\psi_{\lambda} T^{\prime}, T \cap H_{\lambda}^{+}=\phi_{\lambda} T$, and $T \cap H_{\lambda}^{-}=\psi_{\lambda} T$. Set $q=(r+1) / 2$. Since $\mathrm{Z}$ is SL(2) equivariant and homogeneous of degree $2 q+1$, (5) implies that

$$
\mathrm{Z} T+{ }_{p}(1-\lambda)^{q} \psi_{\lambda} \mathrm{Z} T^{\prime}=\lambda^{q} \phi_{\lambda} \mathrm{Z} T+{ }_{p}(1-\lambda)^{q} \psi_{\lambda} \mathrm{Z} T .
$$

1. Let $q \neq 0$. By Lemma 1, Z is a simple valuation. Set $f(x)=h^{p}(\mathrm{Z} T, x)$. Then (7) implies that

$$
f(x)=\lambda^{p q} f\left(\phi_{\lambda}^{t} x\right)+(1-\lambda)^{p q} f\left(\psi_{\lambda}^{t} x\right) \text { for } 0<\lambda<1, x \in \mathbb{R}^{2} .
$$

We need the following lemma, which is also used for the case $q=0$.

Lemma 3. Let $f: \mathbb{R}^{2} \rightarrow \mathbb{R}$ be a function which is positively homogeneous of degree $p$ and for which (8) holds. Then for $x_{1} \geq x_{2} \geq 0$

$$
\begin{aligned}
f\left(x_{1}, x_{2}\right) & =\frac{x_{1}^{p q+p}-x_{2}^{p q+p}}{\left(x_{1}-x_{2}\right)^{p q}} f\left(e_{1}\right), \\
f\left(-x_{1},-x_{2}\right) & =\frac{x_{1}^{p q+p}-x_{2}^{p q+p}}{\left(x_{1}-x_{2}\right)^{p q}} f\left(-e_{1}\right),
\end{aligned}
$$

for $x_{2} \geq x_{1} \geq 0$

$$
\begin{aligned}
f\left(x_{1}, x_{2}\right) & =\frac{x_{2}^{p q+p}-x_{1}^{p q+p}}{\left(x_{2}-x_{1}\right)^{p q}} f\left(e_{2}\right), \\
f\left(-x_{1},-x_{2}\right) & =\frac{x_{2}^{p q+p}-x_{1}^{p q+p}}{\left(x_{2}-x_{1}\right)^{p q}} f\left(-e_{2}\right),
\end{aligned}
$$

and for $x_{1}, x_{2} \geq 0$

$$
\begin{aligned}
& f\left(-x_{1}, x_{2}\right)=\frac{x_{2}^{p q+p}}{\left(x_{1}+x_{2}\right)^{p q}} f\left(e_{2}\right)+\frac{x_{1}^{p q+p}}{\left(x_{1}+x_{2}\right)^{p q}} f\left(-e_{1}\right), \\
& f\left(x_{1},-x_{2}\right)=\frac{x_{1}^{p q+p}}{\left(x_{1}+x_{2}\right)^{p q}} f\left(e_{1}\right)+\frac{x_{2}^{p q+p}}{\left(x_{1}+x_{2}\right)^{p q}} f\left(-e_{2}\right) .
\end{aligned}
$$

Proof. The vector $e_{1}$ is an eigenvector of $\phi_{\lambda}^{t}$ with eigenvalue $\lambda$, and the vector $e_{1}+e_{2}$ is an eigenvector with eigenvalue 1 . The vector $e_{2}$ is an eigenvector of $\psi_{\lambda}^{t}$ with eigenvalue $(1-\lambda)$, and the vector $e_{1}+e_{2}$ is an eigenvector with eigenvalue 1 . Therefore

$$
f\left(e_{1}\right)=\lambda^{p q} f\left(\lambda e_{1}\right)+(1-\lambda)^{p q} f\left(e_{1}+\lambda e_{2}\right)
$$

and

$$
f\left(e_{1}+\lambda e_{2}\right)=\frac{1-\lambda^{p q+p}}{(1-\lambda)^{p q}} f\left(e_{1}\right) .
$$

Since $f$ is homogeneous of degree $p$, this can be written as

$$
f\left(x_{1}, x_{2}\right)=\frac{x_{1}^{p q+p}-x_{2}^{p q+p}}{\left(x_{1}-x_{2}\right)^{p q}} f\left(e_{1}\right)
$$

for $x_{1} \geq x_{2} \geq 0$. Similarly, (10)-(12) are derived.

From (8) we obtain

$$
f\left(-(1-\lambda) e_{1}+\lambda e_{2}\right)=\lambda^{p q+p} f\left(e_{2}\right)+(1-\lambda)^{p q+p} f\left(-e_{1}\right),
$$

which can be written as

$$
f\left(-x_{1}, x_{2}\right)=\frac{x_{2}^{p q+p}}{\left(x_{1}+x_{2}\right)^{p q}} f\left(e_{2}\right)+\frac{x_{1}^{p q+p}}{\left(x_{1}+x_{2}\right)^{p q}} f\left(-e_{1}\right)
$$

for $x_{1}, x_{2} \geq 0$. Similarly, (14) is derived. 
Let $q>1 / p$. If $f\left(e_{1}\right) \neq 0$, then by Lemma 3

$$
\lim _{\lambda \rightarrow 1} f\left(e_{1}+\lambda e_{2}\right)=\lim _{\lambda \rightarrow 1} \frac{1-\lambda^{p q+p}}{(1-\lambda)^{p q}} f\left(e_{1}\right)= \pm \infty .
$$

Since $f(x)=h^{p}(\mathrm{Z} T, x)$ and since $\mathrm{ZT}$ is bounded, this is not possible. Thus $f\left(e_{1}\right)=0$. Similarly, we obtain from Lemma 3 that $f\left(-e_{1}\right)=f\left(e_{2}\right)=f\left(-e_{2}\right)=0$. Therefore Lemma 3 implies that $f(x)=0$ for every $x \in \mathbb{R}^{2}$, that is, ZT $=\{o\}$.

Let $q<1 / p$. Then by Lemma 3 we have

$$
\lim _{\lambda \rightarrow 1} f\left(e_{1}+\lambda e_{2}\right)=\lim _{\lambda \rightarrow 1} f\left(-e_{1}-\lambda e_{2}\right)=0 .
$$

Thus ZTC $\left(e_{1}+e_{2}\right)^{\perp}$ and there are constants $a_{1}, a_{2} \in \mathbb{R}$ such that

$$
f(x)=h^{p}\left(\left[-a_{1}\left(e_{1}-e_{2}\right), a_{2}\left(e_{1}-e_{2}\right)\right], x\right) \text { for } x \in \mathbb{R}^{2} .
$$

If $p>1$ and $q=1 / p-1$, then $a_{1}, a_{2} \geq 0$ and this is just the statement of the proposition. If $q \neq 1 / p-1$, we use (8) with $x= \pm(1,-1)$ and obtain that

$$
a_{i}^{p}=\lambda^{p q+p} a_{i}^{p}+(1-\lambda)^{p q+p} a_{i}^{p} .
$$

This implies that $a_{1}=a_{2}=0$ and $\mathrm{ZT}=\{o\}$.

Let $q=1 / p$. Since $f(x)=h^{p}(\mathrm{ZT}, x)$ is continuous, we obtain from Lemma 3 that $f\left(e_{1}\right)=f\left(e_{2}\right)$ and $f\left(-e_{1}\right)=f\left(-e_{2}\right)$. Thus

$$
\begin{array}{lll}
f\left(x_{1}, x_{2}\right) & =\frac{x_{1}^{p+1}-x_{2}^{p+1}}{x_{1}-x_{2}} f\left(e_{1}\right) & \text { for } x_{1} \geq x_{2} \geq 0, \\
f\left(-x_{1},-x_{2}\right)=\frac{x_{1}^{p+1}-x_{2}^{p+1}}{x_{1}-x_{2}} f\left(-e_{1}\right) & \text { for } x_{1} \geq x_{2} \geq 0, \\
f\left(-x_{1}, x_{2}\right)=\frac{x_{2}^{p+1}}{x_{1}+x_{2}} f\left(e_{1}\right)+\frac{x_{1}^{p+1}}{x_{1}+x_{2}} f\left(-e_{1}\right) & \text { for } x_{1}, x_{2} \geq 0
\end{array}
$$

and $f\left(x_{1}, x_{2}\right)=f\left(x_{2}, x_{1}\right)$. Set $g(x)=\tau h(m(T), x)+h(\mathrm{M} T, x)$ with $\tau \in \mathbb{R}$ for $p=1$ and set $g(x)=h^{p}\left(\mathrm{M}_{p}^{\tau} T, x\right)$ with $-1 \leq \tau \leq 1$ for $p>1$. Then a simple calculation shows that

$$
\begin{array}{ll}
g\left(x_{1}, x_{2}\right)=\frac{(1+\tau)^{p}\left(x_{1}^{p+1}-x_{2}^{p+1}\right)}{(p+1)(p+2)\left(x_{1}-x_{2}\right)} & \text { for } x_{1} \geq x_{2} \geq 0, \\
g\left(x_{1}, x_{2}\right)=\frac{(1-\tau)^{p}\left(x_{1}^{p+1}-x_{2}^{p+1}\right)}{(p+1)(p+2)\left(x_{1}-x_{2}\right)} & \text { for } x_{1} \geq x_{2} \geq 0, \\
g\left(x_{1}, x_{2}\right)=\frac{(1+\tau)^{p} x_{2}^{p+1}+(1-\tau)^{p} x_{1}^{p+1}}{(p+1)(p+2)\left(x_{1}+x_{2}\right)} & \text { for } x_{1}, x_{2} \geq 0
\end{array}
$$

and that $g\left(x_{1}, x_{2}\right)=g\left(x_{2}, x_{1}\right)$. Comparing this with (15) shows that for $p=1$

$$
f(x)=a_{0} h(m(T), x)+a_{1} h(\mathrm{M} T, x),
$$

where $a_{0} \in \mathbb{R}$ and $a_{1} \geq 0$ are suitable constants, and that for $p>1$

$$
f(x)=a h\left(\mathrm{M}_{p}^{\tau} T, x\right),
$$

where $a \geq 0$ and $-1 \leq \tau \leq 1$ are suitable constants. 
2. Let $q=0$. Set $f(x)=h^{p}(\mathrm{Z} T, x)-h^{p}\left(\mathrm{Z} T^{\prime}, x\right)$. By Lemma $1 \mathrm{Z} T^{\prime} \subset e_{1}^{\perp}$. Therefore $\phi_{\lambda} T^{\prime}=T^{\prime}$ and (5) implies that

$$
f(x)=f\left(\phi_{\lambda}^{t} x\right)+f\left(\psi_{\lambda}^{t} x\right) \text { for } 0<\lambda<1, x \in \mathbb{R}^{2} .
$$

Thus (8) holds with $q=0$ and we can apply Lemma 3 .

Since $\mathrm{Z} T^{\prime} \subset e_{1}^{\perp}$, we have $\mathrm{Z} T^{\prime}=\left[-s e_{2}, t e_{2}\right]$ with $s, t \in \mathbb{R},-s \leq t$. Note that if $p>1$, then $h\left(\mathrm{ZT}^{\prime}, \cdot\right) \geq 0$ and $s, t \geq 0$. Setting $a_{0}=f\left(e_{1}\right)+f\left(e_{2}\right), a_{1}=-f\left(e_{2}\right), a_{2}=$ $-f\left(e_{1}\right)+t^{p}$ and $b_{0}=f\left(-e_{1}\right)+f\left(-e_{2}\right), b_{1}=-f\left(-e_{2}\right), b_{2}=-f\left(-e_{1}\right)+s^{p}$, we obtain from Lemma 3 that for $x_{1} \geq x_{2} \geq 0$

$$
\begin{array}{ll}
h^{p}\left(\mathrm{ZT},\left(x_{1}, x_{2}\right)\right) & =x_{1}^{p}\left(a_{0}+a_{1}\right)+x_{2}^{p} a_{2}, \\
h^{p}\left(\mathrm{Z} T,\left(-x_{1},-x_{2}\right)\right) & =x_{1}^{p}\left(b_{0}+b_{1}\right)+x_{2}^{p} b_{2},
\end{array}
$$

for $x_{2} \geq x_{1} \geq 0$

$$
\begin{array}{ll}
h^{p}\left(\mathrm{ZT},\left(x_{1}, x_{2}\right)\right) & =x_{1}^{p} a_{1}+x_{2}^{p}\left(a_{0}+a_{2}\right), \\
h^{p}\left(\mathrm{ZT},\left(-x_{1},-x_{2}\right)\right) & =x_{1}^{p} b_{1}+x_{2}^{p}\left(b_{0}+b_{2}\right),
\end{array}
$$

and for $x_{1}, x_{2} \geq 0$

$$
\begin{aligned}
& h^{p}\left(\mathrm{ZT},\left(-x_{1}, x_{2}\right)\right)=x_{1}^{p}\left(b_{0}+b_{1}\right)+x_{2}^{p}\left(a_{0}+a_{2}\right), \\
& h^{p}\left(\mathrm{ZT},\left(x_{1},-x_{2}\right)\right)=x_{1}^{p}\left(a_{0}+a_{1}\right)+x_{2}^{p}\left(b_{0}+b_{2}\right) .
\end{aligned}
$$

As a support function $h(\mathrm{Z} T, x)$ is convex. Therefore

$$
h\left(\mathrm{ZT}, e_{1}\right)+h\left(\mathrm{ZT}, e_{2}\right) \geq h\left(\mathrm{ZT}, e_{1}+e_{2}\right),
$$

which implies that $a_{0} \geq 0$. Similarly, we obtain $b_{0} \geq 0$. If $p>1$, then $a_{0}+a_{1}, a_{0}+$ $a_{2}, b_{0}+b_{1}, b_{0}+b_{2} \geq 0$. This is just the statement of the proposition. Since

$$
h\left(\mathrm{ZT}, e_{1}+e_{2}\right)+h\left(\mathrm{ZT},-e_{1}+e_{2}\right) \geq 2 h\left(\mathrm{ZT}, e_{2}\right),
$$

we have $a_{1}+b_{0}+b_{1} \geq 0$. Similarly, we obtain $a_{2}+b_{0}+b_{1} \geq 0$ and $a_{0}+a_{1}+b_{1}, a_{0}+$ $a_{1}+b_{2} \geq 0$. Thus the proposition holds true.

Next, we consider the case $n \geq 3$.

Proposition 2. Let $\mathrm{Z}: \mathcal{P}_{o}^{n} \rightarrow \mathcal{K}^{n}, n \geq 3$, be a Minkowski valuation which is $\operatorname{SL}(n)$ equivariant and homogeneous of degree $r$. If $r=n+1$, then there are constants $a_{0} \in \mathbb{R}, a_{1} \geq 0$ such that

$$
\mathrm{Z} T=a_{0} m(T)+a_{1} \mathrm{M} T .
$$

If $r=1$, then there are constants $a, b \geq 0$ such that

$$
\mathrm{Z} T=a T+b(-T) .
$$

In all other cases, $\mathrm{ZT}=\{o\}$.

Proposition $\mathbf{2}_{\mathbf{p}}$. Let $\mathrm{Z}: \mathcal{P}_{o}^{n} \rightarrow \mathcal{K}_{o}^{n}, n \geq 3$, be an $L_{p}$-Minkowski valuation, $p>1$, which is $\mathrm{SL}(n)$ equivariant and homogeneous of degree $r$. If $r=n / p+1$, then there are constants $a \geq 0$ and $-1 \leq \tau \leq 1$ such that

$$
\mathrm{Z} T=a \mathrm{M}_{p}^{\tau} T .
$$

If $r=1$, then there are constants $a, b \geq 0$ such that

$$
\mathrm{Z} T=a T+{ }_{p} b(-T) .
$$

In all other cases, ZT $=\{o\}$.

We prove both propositions at the same time. 
Proof. If $p>1$, let $\mathrm{Z}: \mathcal{P}_{o}^{n} \rightarrow \mathcal{K}_{o}^{n}$, and if $p=1$, let $\mathrm{Z}: \mathcal{P}_{o}^{n} \rightarrow \mathcal{K}^{n}$.

For $0<\lambda<1, i<j$, let $H_{\lambda}=H_{\lambda}(i, j)$ be the hyperplane through $o$ with normal vector $(1-\lambda) e_{i}-\lambda e_{j}$. Then $H_{\lambda}$ dissects $T$ into two simplices $T \cap H_{\lambda}^{+}$and $T \cap H_{\lambda}^{-}$. Since $\mathrm{Z}$ is a valuation, we have

$$
\mathrm{Z} T+{ }_{p} \mathrm{Z}\left(T \cap H_{\lambda}\right)=\mathrm{Z}\left(T \cap H_{\lambda}^{+}\right)+{ }_{p} \mathrm{Z}\left(T \cap H_{\lambda}^{-}\right) .
$$

Define $\phi_{\lambda}=\phi_{\lambda}(i, j), \psi_{\lambda}=\psi_{\lambda}(i, j)$ by

$$
\begin{array}{lll}
\phi_{\lambda} e_{i}=\lambda e_{i}+(1-\lambda) e_{j}, & \phi_{\lambda} e_{k}=e_{k} & \text { for } k \neq i, \\
\psi_{\lambda} e_{j}=\lambda e_{i}+(1-\lambda) e_{j}, & \psi_{\lambda} e_{k}=e_{k} & \text { for } k \neq j .
\end{array}
$$

Then $T \cap H_{\lambda}^{+}=\phi_{\lambda} T$ and $T \cap H_{\lambda}^{-}=\psi_{\lambda} T$. Set $T^{\prime}=T \cap e_{i}^{\perp}$. Then $T \cap H_{\lambda}=\psi_{\lambda} T^{\prime}$. Set $q=(r-1) / n$. Since $\mathrm{Z}$ is $\operatorname{SL}(n)$ equivariant and homogeneous of degree $n q+1$, (17) implies that

$$
\mathrm{Z} T+{ }_{p}(1-\lambda)^{q} \psi_{\lambda} \mathrm{Z} T^{\prime}=\lambda^{q} \phi_{\lambda} \mathrm{Z} T+_{p}(1-\lambda)^{q} \psi_{\lambda} \mathrm{Z} T .
$$

We need the following symmetry relations. Let $i \neq j, k \neq l$ and let $\alpha \in \operatorname{SL}(n)$ be such that $\alpha^{t} e_{i}=e_{k}, \alpha^{t} e_{j}=e_{l}$ and $\alpha T=T$. Since $\mathrm{Z}$ is $\operatorname{SL}(n)$ equivariant, $\mathrm{ZT}=\alpha \mathrm{ZT}$. This implies that for $s, t \in \mathbb{R}$

$$
h\left(\mathrm{ZT}, s e_{i}+t e_{j}\right)=h\left(\mathrm{ZT}, s e_{k}+t e_{l}\right) .
$$

1. Let $q \neq 0$. By Lemma 1, Z is a simple valuation. Set $g(x)=h^{p}(\mathrm{Z} T, x)$. Then (20) implies that

$$
g(x)=\lambda^{p q} g\left(\phi_{\lambda}^{t} x\right)+(1-\lambda)^{p q} g\left(\psi_{\lambda}^{t} x\right) \text { for } 0<\lambda<1, i<j, x \in \mathbb{R}^{n} .
$$

For $k \neq i, j$, we have

$$
g\left(e_{k}\right)=\lambda^{p q} g\left(e_{k}\right)+(1-\lambda)^{p q} g\left(e_{k}\right) .
$$

Let $q \neq 1 / p$. Then this implies that $g\left(e_{k}\right)=0$. Similarly, we obtain that $g\left(-e_{k}\right)=0$. Since (22) implies that Lemma 3 holds for $f(s, t)=g\left(s e_{i}+t e_{j}\right)$, we obtain that $g\left(s e_{i}+t e_{j}\right)=0$ for $(s, t) \in \mathbb{R}^{2}$. Combined with the following lemma this shows that $\mathrm{Z} T=\{o\}$ for $q \neq 1 / p$.

Lemma 4. Let $g_{1}, g_{2}: \mathbb{R}^{n} \rightarrow \mathbb{R}$ be functions for which (22) holds. If $g_{1}(x)=g_{2}(x)$ for every $x \in \mathbb{R}^{n}$ where all but two coordinates vanish, then $g_{1}=g_{2}$.

Proof. Assume that $g_{1}(x)=g_{2}(x)$ holds true for every $x \in \mathbb{R}^{n}$ where at most $k$, $2 \leq k \leq n-1$, coordinates are $\neq 0$. We show that then $g_{1}(x)=g_{2}(x)$ for every $x \in \mathbb{R}^{n}$ where at most $(k+1)$ coordinates are $\neq 0$. So let $x=\left(x_{1}, \ldots, x_{n}\right), n \geq 3$, be such that at most $k$ coordinates are $\neq 0$. Since at least two coordinates of $x$ have the same sign and (22) holds for every pair $i<j$, we may assume that $x_{1}, x_{2}>0$ or $x_{1}, x_{2}<0$ and use (22) for $i=1, j=2$.

First, let $0<x_{2}<x_{1}$ or $x_{1}<x_{2}<0$. By (22), we have for $0<\lambda<1$

$$
g_{i}\left(\psi_{\lambda}^{-t} x\right)=\lambda^{p q} g_{i}\left(\phi_{\lambda}^{t} \psi_{\lambda}^{-t} x\right)+(1-\lambda)^{p q} g_{i}(x) .
$$

Since

$$
\begin{gathered}
\psi_{\lambda}^{-t} x=\left(x_{1},-\frac{\lambda}{1-\lambda} x_{1}-\frac{1}{1-\lambda} x_{2}, x_{3}, \ldots, x_{n}\right), \\
\phi_{\lambda}^{t} \psi_{\lambda}^{-t} x=\left(x_{2},-\frac{\lambda}{1-\lambda} x_{1}-\frac{1}{1-\lambda} x_{2}, x_{3}, \ldots, x_{n}\right),
\end{gathered}
$$

we set $\lambda=x_{2} / x_{1}$ and obtain $0<\lambda<1$. In $\psi_{\lambda}^{-t} x$ and $\phi_{\lambda}^{t} \psi_{\lambda}^{-t} x$ at most $k$ coordinates are $\neq 0$. 
Now, let $0<x_{1}<x_{2}$ or $x_{2}<x_{1}<0$. By (22), we have

$$
g_{i}\left(\phi_{\lambda}^{-t} x\right)=\lambda^{p q} g_{i}(x)+(1-\lambda)^{p q} g_{i}\left(\psi_{\lambda}^{t} \phi_{\lambda}^{-t} x\right) .
$$

Since

$$
\begin{aligned}
\phi_{\lambda}^{-t} x & =\left(\frac{1}{\lambda} x_{1}-\frac{1-\lambda}{\lambda} x_{2}, x_{2}, \ldots, x_{n}\right), \\
\psi_{\lambda}^{t} \phi_{\lambda}^{-t} x & =\left(\frac{1}{\lambda} x_{1}-\frac{1-\lambda}{\lambda} x_{2}, x_{1}, x_{3}, \ldots, x_{n}\right),
\end{aligned}
$$

we set $\lambda=1-x_{1} / x_{2}$ and obtain $0<\lambda<1$. As before we obtain $g_{1}(x)=g_{2}(x)$. In $\phi_{\lambda}^{-t} x$ and $\psi_{\lambda}^{t} \phi_{\lambda}^{-t} x$ at most $k$ coordinates are $\neq 0$.

Let $q=1 / p$. Note that for $x=s e_{1}+t e_{2}, s, t \in \mathbb{R}, h(\mathrm{M} T, x)$ is a multiple of $h\left(\mathrm{M} T_{2},(s, t)\right)$, where $T_{2}$ is the 2-dimensional standard simplex, and that corresponding statements hold for $h(m(T), x)$ and $h\left(\mathrm{M}_{p}^{\tau} T, x\right)$. Set $f(s, t)=g\left(s e_{1}+t e_{2}\right)$. Since (22) implies that Lemma 3 holds for $f(s, t)=g\left(s e_{1}+t e_{2}\right)$, we obtain from (15) and (16) that for $p=1$ and $x=s e_{1}+t e_{2}$,

$$
g(x)=h(\mathrm{Z} T, x)=a_{0} h(m(T), x)+a_{1} h(\mathrm{M} T, x),
$$

where $a_{0} \in \mathbb{R}$ and $a_{1} \geq 0$ are suitable constants. Similarly, we obtain that for $p>1$ and $x=s e_{1}+t e_{2}$,

$$
g(x)=h^{p}(\mathrm{Z} T, x)=a h\left(\mathrm{M}_{p}^{\tau} T, x\right),
$$

where $a \geq 0$ and $-1 \leq \tau \leq 1$ are suitable constants. Since $\mathrm{Z}$ as well as $m, \mathbf{M}, \mathrm{M}_{p}^{\tau}$ are $\mathrm{SL}(n)$ equivariant, we obtain from (21) and the same argument applied to $m, \mathrm{M}, \mathrm{M}_{p}^{\tau}$ that (23) and (24) hold for every $x \in \mathbb{R}^{n}$ where all but two coordinates vanish. Thus applying Lemma 4 shows that the propositions hold true for $q=1 / p$.

2. Let $q=0$. Let $i=1, j=2$. Set $g(x)=h^{p}(\mathrm{Z} T, x)-h^{p}\left(\mathrm{Z} T^{\prime}, x\right)$. By Lemma 1 Z $T^{\prime} \subset e_{1}^{\perp}$. Therefore $\phi_{\lambda} T^{\prime}=T^{\prime}$ and (17) implies that

$$
g(x)=g\left(\phi_{\lambda}^{t} x\right)+g\left(\psi_{\lambda}^{t} x\right) \text { for } 0<\lambda<1, x \in \mathbb{R}^{n} .
$$

Thus (22) holds with $q=0$. Set $a=h^{p}\left(\mathrm{Z} T^{\prime}, e_{2}\right)$ and $b=h^{p}\left(\mathrm{Z} T^{\prime},-e_{2}\right)$. We apply Lemma 3 with $f\left(x_{1}, x_{2}\right)=h^{p}(\mathrm{ZT}, x)-h^{p}\left(\mathrm{Z} T^{\prime}, x\right)$ for $x=x_{1} e_{1}+x_{2} e_{2}$ and obtain that for $x_{1} \geq x_{2} \geq 0$

$$
\begin{aligned}
& h^{p}\left(\mathrm{ZT},\left(x_{1}, x_{2}, 0, \ldots, 0\right)\right) \\
& h^{p}\left(\mathrm{ZT},\left(-x_{1},-x_{2}, 0, \ldots, 0\right)\right)=\left(x_{1}^{p}-x_{2}^{p}\right) f\left(e_{1}\right)+x_{2}^{p} a, \\
& =\left(x_{1}^{p}-x_{2}^{p}\right) f\left(-e_{1}\right)+x_{2}^{p} b,
\end{aligned}
$$

for $x_{2} \geq x_{1} \geq 0$

$$
\begin{aligned}
& h^{p}\left(\mathrm{ZT},\left(x_{1}, x_{2}, 0, \ldots, 0\right)\right) \\
& h^{p}\left(\mathrm{ZT},\left(-x_{1},-x_{2}, 0, \ldots, 0\right)\right)=\left(x_{2}^{p}-x_{1}^{p}\right) f\left(e_{2}\right)+x_{2}^{p} a, \\
& =\left(x_{2}^{p}-x_{1}^{p}\right) f\left(-e_{2}\right)+x_{2}^{p} b,
\end{aligned}
$$

and for $x_{1}, x_{2} \geq 0$

$$
\begin{aligned}
& h^{p}\left(\mathrm{ZT},\left(-x_{1}, x_{2}, 0, \ldots, 0\right)\right)=x_{2}^{p} f\left(e_{2}\right)+x_{1}^{p} f\left(-e_{1}\right)+x_{2} a, \\
& h^{p}\left(\mathrm{ZT},\left(x_{1},-x_{2}, 0, \ldots, 0\right)\right)=x_{1}^{p} f\left(e_{1}\right)+x_{2}^{p} f\left(-e_{2}\right)+x_{2} b .
\end{aligned}
$$

Note that (21), (25), and (26) imply that

$$
f\left(e_{1}\right)=f\left(e_{2}\right)+a .
$$

Since there is a $\beta \in \mathrm{SL}(n)$ such that $\beta e_{2}=e_{3}, \beta e_{3}=e_{2}$, and $\beta T^{\prime}=T^{\prime}$ and since $\mathrm{Z}$ is $\mathrm{SL}(n)$ equivariant, we have

$$
h\left(\mathrm{Z} T^{\prime}, e_{2}\right)=h\left(\mathrm{Z} T^{\prime}, e_{3}\right) .
$$


Since $e_{3}$ and $e_{1}+e_{2}$ are eigenvectors with eigenvalue 1 of $\phi_{\lambda}^{t}$ and $\psi_{\lambda}^{t}$, it follows from (20) that

$$
h\left(\mathrm{Z} T^{\prime}, e_{3}\right)=h\left(\mathrm{ZT}, e_{3}\right) \text { and } h\left(\mathrm{Z} T^{\prime}, e_{1}+e_{2}\right)=h\left(\mathrm{ZT}, e_{1}+e_{2}\right) .
$$

By (29), (21), and (30), we obtain

$$
a=h^{p}\left(\mathrm{Z} T^{\prime}, e_{2}\right)=h^{p}\left(\mathrm{ZT}, e_{1}\right)=f\left(e_{1}\right)
$$

and therefore by (28) that $f\left(e_{2}\right)=0$. Similarly, we get $b=f\left(-e_{1}\right)$. Thus it follows from (25)-(27) that

$$
h^{p}(\mathrm{Z} T, x)=a h^{p}(T, x)+b h^{p}(-T, x)
$$

for every $x=\left(x_{1}, x_{2}, 0, \ldots, 0\right)$. Since $\mathrm{ZT}$ is convex, we have $a, b \geq 0$.

The operators $\mathrm{Z}$ and $P \mapsto a P+{ }_{p} b(-P)$ are $\mathrm{SL}(n)$ equivariant. Therefore (31) holds for every $x \in \mathbb{R}^{n}$ where all but two coordinates vanish. We apply Lemma 4 with

$$
g_{1}(x)=h^{p}(\mathrm{Z} T, x)-h^{p}\left(\mathrm{Z} T^{\prime}, x\right)
$$

and

$$
g_{2}(x)=a\left(h^{p}(T, x)-h^{p}\left(T^{\prime}, x\right)\right)+b\left(h^{p}(-T, x)-h^{p}\left(-T^{\prime}, x\right)\right)
$$

and obtain that for every $x \in \mathbb{R}^{n}$

$$
h^{p}(\mathrm{Z} T, x)=a h^{p}(T, x)+b h^{p}(-T, x)+w(x),
$$

where

$$
w(x)=h^{p}\left(\mathrm{Z} T^{\prime}, x\right)-a h^{p}\left(T^{\prime}, x\right)-b h^{p}\left(-T^{\prime}, x\right) .
$$

We identify $e_{1}^{\perp}$ with $\mathbb{R}^{n-1}$ and use induction on the dimension to obtain $Z T^{\prime}$. The operator $\mathrm{Z}$ restricted to convex polytopes in $e_{1}^{\perp}$ is a homogeneous, $\operatorname{SL}(n-1)$ equivariant $L_{p}$-Minkowski valuation. Let $n=3$. Then we obtain from Propositions 1 and 1 that there are constants such that

$$
\mathrm{Z} T^{\prime}=a_{1} T^{\prime}+{ }_{p} b_{1}\left(-T^{\prime}\right)+{ }_{p} a_{2}\left[o, e_{2}\right]+{ }_{p} b_{2}\left[o,-e_{2}\right]+{ }_{p} a_{3}\left[o, e_{3}\right]+{ }_{p} b_{3}\left[o,-e_{3}\right] .
$$

We have

$$
h^{p}\left(\mathrm{ZT}, e_{2}+e_{3}\right)=a+w\left(e_{2}+e_{3}\right)=h^{p}\left(\mathrm{ZT}^{\prime}, e_{2}+e_{3}\right)=a_{1}+a_{2}+a_{3}
$$

and

$$
h^{p}\left(\mathrm{ZT}, e_{1}+e_{3}\right)=a+w\left(e_{1}+e_{2}\right)=h^{p}\left(\mathrm{Z} T^{\prime}, e_{2}\right)=a_{1}+a_{2} .
$$

Thus (21) implies that $a_{3}=0$. Similarly, we obtain $a_{2}=b_{2}=b_{3}=0$. This shows that $w(x)=0$ for $n=3$ and $\mathrm{ZT}=a T+{ }_{p} b(-T)$. Let $n \geq 4$. Then we get by induction that

$$
\mathrm{Z} T^{\prime}=a T^{\prime}+{ }_{p} b\left(-T^{\prime}\right) .
$$

Therefore $w(x)=0$ also in this case. Thus ZT $=a T+{ }_{p} b(-T)$ holds for $n \geq 4$. 


\section{Contravariant Minkowski valuations}

In this section, our main result on the classification of $\mathrm{SL}(n)$ contravariant Minkowski valuations is formulated. The most important example is the projection operator $\Pi: \mathcal{K}^{n} \rightarrow \mathcal{K}^{n}$. The following transformation rule for $\Pi$, which is due to Petty (cf. [5]), shows that $\Pi$ is $\mathrm{SL}(n)$ contravariant and homogeneous of degree $(n-1)$ :

$$
\Pi(\phi K)=|\operatorname{det} \phi| \phi^{-t} \Pi K \quad \text { for } \phi \in \mathrm{GL}(n) .
$$

On $\mathcal{P}_{o}^{n}$ we define an additional operator with the same transformation behaviour. Note that for a polytope $P, \Pi P$ can be written in the following way (cf. [5]). A vector $v$ is a scaled facet normal of $P$, if $v$ is an outer normal to a facet of $P$ and if the length of $v$ is equal to the $(n-1)$-dimensional volume of the corresponding facet. Then

$$
\Pi P=\sum_{v \in \mathcal{V}(P)}[o, v]
$$

where $\mathcal{V}(P)$ is the set of scaled facet normals of $P$. Define the operator $\Pi_{o}: \mathcal{P}_{o}^{n} \rightarrow$ $\mathcal{K}^{n}$ by

$$
\Pi_{o} P=\sum_{v \in \mathcal{V}_{o}(P)}[o, v],
$$

where $\mathcal{V}_{o}(P)$ is the set of scaled facet normals of $P$ that correspond to facets that contain the origin. We set $\Pi_{o} P=\{o\}$ if $P$ contains the origin in its interior. Note that $\Pi_{o}$ is a Minkowski valuation and that it is not continuous. For $a_{1} \geq 0$, $a_{2}, a_{3} \in \mathbb{R}$ with $a_{1}+a_{2}+a_{3} \geq 0$, the operator $\mathrm{Z}: \mathcal{P}_{o}^{n} \rightarrow \mathcal{K}^{n}$ defined by

$$
\mathrm{Z} P=a_{1} \Pi P+a_{2} \Pi_{o} P+a_{3}\left(-\Pi_{o} P\right)
$$

is a Minkowski valuation which is $\operatorname{SL}(n)$ contravariant and homogeneous of degree $(n-1)$. As we will see, for $n \geq 3$ these are already all examples of homogeneous, $\mathrm{SL}(n)$ contravariant Minkowski valuations on $\mathcal{P}_{o}^{n}$.

For $n=2$, we have additional examples. Let $\psi_{\pi / 2}$ denote the rotation by an angle $\pi / 2$. If $\mathrm{Z}: \mathcal{P}_{o}^{2} \rightarrow \mathcal{K}^{2}$ is $\mathrm{SL}(2)$ equivariant, then

$$
\left(\psi_{\pi / 2} \mathrm{Z}\right)(\phi P)=\psi_{\pi / 2} \phi \psi_{\pi / 2}^{-1}\left(\psi_{\pi / 2} \mathrm{Z}\right) P=\phi^{-t}\left(\psi_{\pi / 2} \mathrm{Z}\right) P,
$$

that is, $\psi_{\pi / 2} \mathrm{Z}$ is $\mathrm{SL}(2)$ contravariant. If $\mathrm{Z}$ is a Minkowski valuation and homogeneous of degree $r$, so is $\psi_{\pi / 2} \mathrm{Z}$. This implies that the rotated versions of the operators from Theorem 1 are homogeneous, SL(2) contravariant Minkowski valuations.

The following result is our classification of $\operatorname{SL}(n)$ contravariant Minkowski valuations. The proof is given in Section 6 .

Theorem 2. Let $\mathrm{Z}: \mathcal{P}_{o}^{n} \rightarrow \mathcal{K}^{n}, n \geq 3$, be a Minkowski valuation which is $\mathrm{SL}(n)$ contravariant and homogeneous of degree $r$. If $r=n-1$, then there are constants $a_{1} \geq 0, a_{2}, a_{3} \in \mathbb{R}$ with $a_{1}+a_{2}+a_{3} \geq 0$ such that

$$
\mathrm{Z} P=a_{1} \Pi P+a_{2} \Pi_{o} P+a_{3}\left(-\Pi_{o} P\right)
$$

for every $P \in \mathcal{P}_{o}^{n}$. In all other cases, $\mathrm{Z} P=\{o\}$ for every $P \in \mathcal{P}_{o}^{n}$.

Let $\mathrm{Z}: \mathcal{P}_{o}^{2} \rightarrow \mathcal{K}^{2}$ be a Minkowski valuation which is $\mathrm{SL}(2)$ contravariant and homogeneous of degree $r$. If $r=3$, then there are constants $a_{0} \in \mathbb{R}$ and $a_{1} \geq 0$ such that

$$
\mathrm{Z} P=\psi_{\pi / 2}\left(a_{0} m(P)+a_{1} \mathrm{M} P\right)
$$


for every $P \in \mathcal{P}_{o}^{2}$. If $r=1$, then there are constants $a_{0}, b_{0} \geq 0$ and $a_{i}, b_{i} \in \mathbb{R}$ with $a_{i}+b_{0}+b_{1}, a_{0}+a_{1}+b_{i} \geq 0, i=1,2$, such that

$$
\mathrm{Z} P=\psi_{\pi / 2}\left(a_{0} P+b_{0}(-P)+\sum\left(a_{i} E_{i}+b_{i}\left(-E_{i}\right)\right)\right)
$$

for every $P \in \mathcal{P}_{o}^{2}$, where the sum is taken over $E_{i} \in \mathcal{E}_{o}(P)$. In all other cases, $\mathrm{Z} P=\{o\}$ for every $P \in \mathcal{P}_{o}^{2}$.

The following simple consequence of this theorem holds.

Corollary 2.1. If $\mathrm{Z}: \mathcal{K}_{o}^{n} \rightarrow \mathcal{K}^{n}, n \geq 3$, is a continuous, homogeneous, $\operatorname{SL}(n)$ contravariant Minkowski valuation, then there is a constant $a \geq 0$ such that

$$
\mathrm{Z} K=a \Pi K
$$

for every $K \in \mathcal{K}_{o}^{n}$.

If $\mathrm{Z}: \mathcal{K}_{o}^{2} \rightarrow \mathcal{K}^{2}$ is a continuous, homogeneous, $\mathrm{SL}(2)$ contravariant Minkowski valuation, then there are constants $a_{0} \in \mathbb{R}$ and $a_{1}, b_{1} \geq 0$ such that

$$
\mathrm{Z} K=\psi_{\pi / 2}\left(a_{0} m(K)+a_{1} \mathrm{M} K\right) \quad \text { or } \quad \mathrm{Z} K=\psi_{\pi / 2}\left(a_{1} K+b_{1}(-K)\right)
$$

for every $K \in \mathcal{K}_{o}^{2}$.

For translation invariant valuations we obtain the following result. The proof is given in Section 7

Corollary 2.2. Let $\mathrm{Z}: \mathcal{P}^{n} \rightarrow \mathcal{K}^{n}, n \geq 2$, be a translation invariant, $\operatorname{SL}(n)$ contravariant Minkowski valuation. Then there is a constant $c \geq 0$ such that $\mathrm{Z}=$ $c \Pi$.

\section{Contravariant $L_{p}$-Minkowski valuations}

In [25] Lutwak, Yang, and Zhang introduced the $L_{p}$-projection operator $\Pi_{p}$ and obtained an $L_{p}$-version of the Petty projection inequality. For $K \in \mathcal{K}_{o}^{n}$ which contain the origin as an interior point, $\Pi_{p}$ is defined by

$$
h^{p}\left(\Pi_{p} K, v\right)=c_{n, p} \int_{S^{n-1}}|v \cdot u|^{p} d S_{p}(K, u)
$$

for $v \in \mathbb{R}^{n}$, where $c_{n, p}$ is chosen such that $\Pi_{p} B=B$ for the unit ball $B$. Here $S_{p}(K, \cdot)$ is the $L_{p}$-surface area measure of $K$, that is, $S_{p}(K, \cdot)=h(K, \cdot)^{1-p} S(K, \cdot)$, where $S(K, \cdot)$ is the surface area measure of $K$. It is proved in [25] that

$$
\Pi_{p}(\phi K)=|\operatorname{det} \phi|^{1 / p} \phi^{-t} \Pi_{p} K \text { for } \phi \in \mathrm{GL}(n) .
$$

Note that $\Pi_{p}$ is an $L_{p}$-Minkowski valuation on convex bodies that contain the origin in their interiors and that it is not bounded on $\mathcal{K}_{o}^{n}$. We need the following generalization of this operator. For $-1 \leq \tau \leq 1$ and $K \in \mathcal{K}_{o}^{n}$ which contain $o$ as an interior point, define $\Pi_{p}^{\tau} K$ by

$$
h^{p}\left(\Pi_{p}^{\tau} K, v\right)=c_{n, p} \int_{S^{n-1}}(|v \cdot u|+\tau(v \cdot u))^{p} d S_{p}(K, u) .
$$

Then $\Pi_{p}^{\tau}$ is an $\mathrm{SL}(n)$ contravariant $L_{p}$-Minkowski valuation on convex bodies that contain the origin in their interiors. For $P \in \mathcal{P}_{o}^{n}$, set

$$
h^{p}\left(\hat{\Pi}_{p}^{\tau} P, v\right)=c_{n, p} \int_{S^{n-1} \backslash \omega_{o}(P)}(|v \cdot u|+\tau(v \cdot u))^{p} d S_{p}(P, u),
$$


where $\omega_{o}(P)$ is the set of outer unit normal vectors to facets of $P$ that contain the origin. Note that $\hat{\Pi}_{p}^{\tau}$ is bounded but not continuous on $\mathcal{K}_{o}^{n}$ and that it is an $L_{p}$-Minkowski valuation. As we will show, these operators are the only examples of homogeneous, $\mathrm{SL}(n)$ contravariant $L_{p}$-Minkowski valuations for $n \geq 3$. For $n=2$, the rotated version of the operators from Theorem 1, are additional examples.

The proof of the following result is given in Section 6 .

Theorem $\mathbf{2}_{\mathbf{p}}$. Let $\mathrm{Z}: \mathcal{P}_{o}^{n} \rightarrow \mathcal{K}^{n}, n \geq 3$, be an $L_{p}$-Minkowski valuation, $p>1$, which is $\mathrm{SL}(n)$ contravariant and homogeneous of degree $r$. If $r=n / p-1$, then there are constants $a \geq 0$ and $-1 \leq \tau \leq 1$ such that

$$
\mathrm{Z} P=a \hat{\Pi}_{p}^{\tau} P
$$

for every $P \in \mathcal{P}_{o}^{n}$. In all other cases, $\mathrm{Z} P=\left\{\right.$ o for every $P \in \mathcal{P}_{o}^{n}$.

Let $\mathrm{Z}: \mathcal{P}_{o}^{2} \rightarrow \mathcal{K}^{2}$ be an $L_{p}$-Minkowski valuation, $p>1$, which is $\mathrm{SL}(2)$ contravariant and homogeneous of degree $r$. If $r=2 / p+1$, then there are constants $a \geq 0$ and $-1 \leq \tau \leq 1$ such that

$$
\mathrm{Z} P=a \psi_{\pi / 2} \mathrm{M}_{p}^{\tau} P
$$

for every $P \in \mathcal{P}_{o}^{2}$. If $r=1$, then there are constants $a_{0}, b_{0} \geq 0$ and $a_{i}, b_{i} \in \mathbb{R}$ with $a_{0}+a_{i}, b_{0}+b_{i} \geq 0, i=1,2$, such that

$$
\mathrm{Z} P=\psi_{\pi / 2}\left(a_{0} P+{ }_{p} b_{0}(-P)+{ }_{p} \sum^{p}\left(a_{i} E_{i}+{ }_{p} b_{i}\left(-E_{i}\right)\right)\right)
$$

for every $P \in \mathcal{P}_{o}^{2}$, where the sum is taken over $E_{i} \in \mathcal{E}_{o}(P)$. If $r=2 / p-1$, then there are constants $a \geq 0$ and $-1 \leq \tau \leq 1$ such that

$$
\mathrm{Z} P=a \hat{\Pi}_{p}^{\tau} P
$$

for every $P \in \mathcal{P}_{o}^{2}$. In all other cases, $\mathrm{Z} P=\{$ o $\}$ for every $P \in \mathcal{P}_{o}^{2}$.

As a simple consequence we obtain the following result.

Corollary. If $\mathrm{Z}: \mathcal{K}_{o}^{n} \rightarrow \mathcal{K}^{n}, n \geq 3$, is a continuous, homogeneous, $\mathrm{SL}(n)$ contravariant $L_{p}$-Minkowski valuation, $p>1$, then

$$
\mathrm{Z} K=\{o\}
$$

for every $K \in \mathcal{K}_{o}^{n}$.

If $\mathrm{Z}: \mathcal{K}_{o}^{2} \rightarrow \mathcal{K}^{2}$ is a continuous, homogeneous, $\mathrm{SL}(2)$ contravariant $L_{p}$-Minkowski valuation, $p>1$, then there are constants $a, b \geq 0$ and $-1 \leq \tau \leq 1$ such that

$$
\mathrm{Z} K=a \psi_{\pi / 2} \mathrm{M}_{p}^{\tau} K \quad \text { or } \quad \mathrm{Z} K=\psi_{\pi / 2}\left(a K+{ }_{p} b(-K)\right)
$$

for every $K \in \mathcal{K}_{o}^{2}$.

\section{Proof of Theorems 2 and 2 p}

As a first step in the proof, we show that every operator which is $\operatorname{SL}(n)$ contravariant and homogeneous of degree $r \neq n-1$ is simple.

Lemma 5. Let $\mathrm{Z}: \mathcal{P}_{o}^{n} \rightarrow \mathcal{K}^{n}$ be an operator which is $\mathrm{SL}(n)$ contravariant and homogeneous of degree $r$. If $\operatorname{dim} P<(n-1)$, then $\mathrm{Z} P=\{o\}$. If $\operatorname{dim} P=(n-1)$, then $\mathrm{Z} P \subset(\operatorname{lin} P)^{\perp}$. If $\operatorname{dim} P=(n-1)$ and $r \neq n-1$, then $\mathrm{Z} P=\{o\}$. 
Proof. Let $P \in \mathcal{P}_{o}^{n}$ be such that $\operatorname{lin} P$ is the $k$-dimensional subspace with equation $x_{k+1}=\ldots=x_{n}=0$. Since every $P^{\prime} \in \mathcal{P}_{o}^{n}$ with $\operatorname{dim} P^{\prime}=k$ is a linear image of such a polytope $P$ and since $\mathrm{Z}$ is $\mathrm{SL}(n)$ contravariant, it suffices to prove the lemma in this case. Let

$$
\phi=\left(\begin{array}{cc}
I & B \\
0 & A
\end{array}\right)
$$

where $I$ is the $k \times k$ identity matrix, 0 is the $(n-k) \times k$ zero matrix, $B$ is an $(n-k) \times k$ matrix, and $A$ is an $(n-k) \times(n-k)$ matrix with determinant 1 . Then $\phi \in \mathrm{SL}(n)$,

$$
\phi^{-t}=\left(\begin{array}{cc}
I & 0 \\
C & A^{-t}
\end{array}\right)
$$

with $C=-A^{-t} B^{t}$, and

$$
\phi P=P .
$$

Write $x=\left(\begin{array}{c}x^{\prime} \\ x^{\prime \prime}\end{array}\right)$ with $x^{\prime}=\left(x_{1}, \ldots, x_{k}\right)$ and $x^{\prime \prime}=\left(x_{k+1}, \ldots, x_{n}\right)$ for $x \in \mathbb{R}^{n}$. Let $x \in \mathrm{Z} P$. Since $\mathrm{Z}$ is $\mathrm{SL}(n)$ contravariant, (32) implies that $y=\phi^{-t} x \in \mathrm{Z} P$, that is,

$$
\left(\begin{array}{c}
y^{\prime} \\
y^{\prime \prime}
\end{array}\right)=\left(\begin{array}{c}
x^{\prime} \\
C x^{\prime}+A^{-t} x^{\prime \prime}
\end{array}\right) \in \mathrm{ZP} .
$$

This is true for every $(n-k) \times k$ matrix $B$ and every $(n-k) \times(n-k)$ matrix $A$ with determinant 1 . If $x^{\prime} \neq o^{\prime}$, this implies that $y^{\prime \prime}$ can be an arbitrary vector. Since Z $P$ is bounded, it follows that $x^{\prime}=o^{\prime}$. Thus Z $P \subset(\operatorname{lin} P)^{\perp}$. If $k=(n-1)$ and $q=1$, this proves the lemma. Let $k<(n-1)$. Then $x^{\prime}=o^{\prime}$ and (33) holds for every $(n-k) \times(n-k)$ matrix $A$ with determinant 1 . Since $\mathrm{Z} P$ is bounded and $(n-k) \geq 2$, this implies that $x^{\prime \prime}=o^{\prime \prime}$. Let $k=(n-1), r \neq n-1$, and let

$$
\phi=\left(\begin{array}{ll}
I & 0 \\
0 & s
\end{array}\right)
$$

where $I$ is the $(n-1) \times(n-1)$ identity matrix and $s \geq 0$. Then

$$
\mathrm{Z}(\phi P)=s^{(r-(n-1)) / n} \mathrm{Z} P=\mathrm{Z} P .
$$

Since this holds for every $s \geq 0$ and $\mathrm{Z} P$ is bounded, this implies that $\mathrm{Z} P=\{o\}$.

By Lemmas 2 and 2 , it suffices to determine the value of $\mathrm{Z}$ for $n$-dimensional simplices to prove Theorems 2 and 2 . Since Z is $\operatorname{SL}(n)$ contravariant and homogeneous, it is enough to determine $\mathrm{Z} T$, where $T$ is the simplex with vertices $o, e_{1}, \ldots, e_{n}$.

We start with the planar case.

Proposition 3. Let $\mathrm{Z}: \mathcal{P}_{o}^{2} \rightarrow \mathcal{K}^{2}$ be a Minkowski valuation which is $\mathrm{SL}(2)$ contravariant and homogeneous of degree $r$. If $r=3$, then there are constants $a_{0} \in \mathbb{R}$ and $a_{1} \geq 0$ such that

$$
\mathrm{ZT}=\psi_{\pi / 2}\left(a_{0} m(T)+a_{1} \mathrm{M} T\right) .
$$

If $r=1$, then there are constants $a_{0}, b_{0} \geq 0$ and $a_{i}, b_{i} \in \mathbb{R}$ with $a_{i}+b_{0}+b_{1}, a_{0}+$ $a_{2}+b_{i} \geq 0, i=1,2$, such that

$$
\mathrm{ZT}=\psi_{\pi / 2}\left(a_{0} T+b_{0}(-T)+a_{1}\left[o, e_{1}\right]+b_{1}\left[o,-e_{1}\right]+a_{2}\left[o, e_{2}\right]+b_{2}\left[o,-e_{2}\right]\right) .
$$

In all other cases, $\mathrm{ZT}=\{o\}$. 
Proposition $3_{\mathrm{p}}$. Let $\mathrm{Z}: \mathcal{P}_{o}^{2} \rightarrow \mathcal{K}_{o}^{2}$ be an $L_{p}$-Minkowski valuation, $p>1$, which is $\mathrm{SL}(2)$ contravariant and homogeneous of degree $r$. If $r=2 / p+1$, then there are constants $a \geq 0$ and $-1 \leq \tau \leq 1$ such that

$$
\mathrm{ZT}=a \psi_{\pi / 2} \mathrm{M}_{p}^{\tau} T
$$

If $r=1$, then there are constants $a_{0}, b_{0} \geq 0$ and $a_{i}, b_{i} \in \mathbb{R}$ with $a_{0}+a_{i}, b_{0}+b_{i} \geq 0$, $i=1,2$, such that

$\mathrm{ZT}=\psi_{\pi / 2}\left(a_{0} T+{ }_{p} b_{0}(-T)+_{p} a_{1}\left[o, e_{1}\right]+{ }_{p} b_{1}\left[o,-e_{1}\right]+{ }_{p} a_{2}\left[o, e_{2}\right]+_{p} b_{2}\left[o,-e_{2}\right]\right)$.

If $r=2 / p-1$, then there are constants $a_{1}, a_{2} \geq 0$ such that

$$
\mathrm{ZT}=\left[-a_{1}\left(e_{1}+e_{2}\right), a_{2}\left(e_{1}+e_{2}\right)\right] .
$$

In all other cases, $\mathrm{Z} T=\{o\}$.

That these propositions hold true can be seen in the following way. Let $\mathrm{Z}$ be as in Proposition 3 or 3, Then $\psi_{\pi / 2} \mathrm{Z}$ is an $L_{p}$-Minkowski valuation which is $\operatorname{SL}(2)$ equivariant and homogeneous of degree $r$. Thus Propositions 1 and 1 , immediately imply that the above propositions hold true.

Next, we consider the case $n \geq 3$.

Proposition 4. Let $\mathrm{Z}: \mathcal{P}_{o}^{n} \rightarrow \mathcal{K}^{n}, n \geq 3$, be a Minkowski valuation, which is $\mathrm{SL}(n)$ contravariant and homogeneous of degree $r$. If $r=n-1$, then there are constants $a_{1} \geq 0, a_{2}, a_{3} \in \mathbb{R}$ with $a_{1}+a_{2}+a_{3} \geq 0$ such that

$$
\mathrm{ZT}=a_{1} \Pi T+a_{2} \Pi_{o} T+a_{3}\left(-\Pi_{o} T\right) .
$$

In all other case, $\mathrm{ZT}=\{o\}$.

Proposition $4_{\mathrm{p}}$. Let $\mathrm{Z}: \mathcal{P}_{o}^{n} \rightarrow \mathcal{K}_{o}^{n}, n \geq 3$, be an $L_{p}$-Minkowski valuation, $p>1$, which is $\mathrm{SL}(n)$ contravariant and homogeneous of degree $r$. If $r=n / p-1$, then there are constants $a_{1}, a_{2} \geq 0$, such that

$$
\mathrm{ZT}=\left[-a_{1}\left(e_{1}+\ldots+e_{n}\right), a_{2}\left(e_{1}+\ldots+e_{n}\right)\right] .
$$

In all other cases, $\mathrm{ZT}=\{o\}$.

We prove both propositions at the same time.

Proof. If $p>1$, let $\mathrm{Z}: \mathcal{P}_{o}^{n} \rightarrow \mathcal{K}_{o}^{n}$, and if $p=1$, let $\mathrm{Z}: \mathcal{P}_{o}^{n} \rightarrow \mathcal{K}^{n}$.

For $0<\lambda<1, i<j$, let $H_{\lambda}=H_{\lambda}(i, j)$ be the hyperplane through $o$ with normal vector $(1-\lambda) e_{i}-\lambda e_{j}$. Then $H_{\lambda}$ dissects $T$ into the two simplices $\phi_{\lambda} T$ and $\psi_{\lambda} T$, where $\phi_{\lambda}$ and $\psi_{\lambda}$ are defined by (18). Set $T^{\prime}=T \cap e_{i}^{\perp}$. Then $T \cap H_{\lambda}=\psi_{\lambda} T^{\prime}$. Set $q=(r+1) / n$. Since $\mathrm{Z}$ is $\mathrm{SL}(n)$ contravariant, homogeneous of degree $n q-1$ and an $L_{p}$-Minkowski valuation, we have

$$
\mathrm{ZT}+{ }_{p}(1-\lambda)^{q} \psi_{\lambda}^{-t} \mathrm{Z} T^{\prime}=\lambda^{q} \phi_{\lambda}^{-t} \mathrm{Z} T+{ }_{p}(1-\lambda)^{q} \psi_{\lambda}^{-t} \mathrm{Z} T .
$$

Denote by $\alpha_{\pi / 2}=\alpha_{\pi / 2}(i, j)$ the rotation by an angle $\pi / 2$ in the plane $\operatorname{lin}\left\{e_{i}, e_{j}\right\}$, that is,

$$
\alpha_{\pi / 2} e_{i}=e_{j}, \quad \alpha_{\pi / 2} e_{j}=-e_{i}, \quad \alpha_{\pi / 2} e_{k}=e_{k} \quad \text { for } k \neq i, j .
$$

Then we obtain that

$$
\left(\alpha_{\pi / 2} \mathrm{Z}\right)\left(\phi_{\lambda} T\right)=\lambda^{(r+1) / n} \alpha_{\pi / 2} \phi_{\lambda}^{-t} \alpha_{\pi / 2}^{-1}\left(\alpha_{\pi / 2} \mathrm{Z}\right) T=\lambda^{(r-1) / n} \phi_{\lambda}\left(\alpha_{\pi / 2} \mathrm{Z}\right) T
$$

and

$$
\left(\alpha_{\pi / 2} \mathrm{Z}\right)\left(\psi_{\lambda} T\right)=\lambda^{(r-1) / n} \psi_{\lambda}\left(\alpha_{\pi / 2} \mathrm{Z}\right) T
$$


1. Let $q \neq 1$. By Lemma 1, Z is a simple valuation. Set $g(x)=h^{p}\left(\alpha_{\pi / 2} \mathrm{Z} T, x\right)$. Then (34)-(36) imply that

$$
g(x)=\lambda^{p q} g\left(\phi_{\lambda}^{t} x\right)+(1-\lambda)^{p q} g\left(\psi_{\lambda}^{t} x\right) \text { for } 0<\lambda<1, i<j, x \in \mathbb{R}^{n} .
$$

Therefore we have for $k \neq i, j$

$$
g\left(e_{k}\right)=\lambda^{p q} g\left(e_{k}\right)+(1-\lambda)^{p q} g\left(e_{k}\right) .
$$

If $q \neq 1 / p$, this implies that $g\left(e_{k}\right)=0$. Similarly, we obtain that $g\left(-e_{k}\right)=0$. Since (37) implies that Lemma 3 holds for $f\left(x_{1}, x_{2}\right)=g\left(x_{1} e_{i}+x_{2} e_{j}\right)$, we obtain that $g(x)=0$ for $x=x_{1} e_{i}+x_{2} e_{j}$. Thus Lemma 团implies that ZT $=\{o\}$.

If $q=1 / p$, then (37) implies that

$$
g\left(e_{i}-e_{j}\right)=\lambda^{1-p} g\left(e_{i}-e_{j}\right)+(1-\lambda)^{1-p} g\left(e_{i}-e_{j}\right) .
$$

Therefore $g\left(e_{i}-e_{j}\right)=0$. Similarly, we obtain that $g\left(e_{j}-e_{i}\right)=0$. Thus we have for $i<j$,

$$
\mathrm{ZT} \subset\left(e_{i}-e_{j}\right)^{\perp} .
$$

This shows that ZT $=\left[-a_{1}\left(e_{1}+\ldots+e_{n}\right), a_{2}\left(e_{1}+\ldots+e_{n}\right)\right]$ with suitable constants $a_{1}, a_{2} \geq 0$.

2. Let $q=1$. Lemma 5 implies that $\mathrm{Z} T^{\prime} \subset \operatorname{lin}\left\{e_{i}\right\}$. Since there is a $\beta \in \operatorname{SL}(n)$ such that $\beta e_{i}=-e_{i}$ and $\beta T^{\prime}=T^{\prime}$ and since $\mathrm{Z}$ is $\mathrm{SL}(n)$ contravariant, we obtain that $\mathrm{ZT}^{\prime}=\left[-a e_{i}, a e_{i}\right]$ with $a \geq 0$. By (34) we have for $k \neq i, j$

$h^{p}\left(\mathrm{ZT}, e_{k}\right)+(1-\lambda)^{p} h^{p}\left(\mathbf{Z} T^{\prime}, e_{k}\right)=h^{p}\left(\mathbf{Z} T, e_{k}\right)=\lambda^{p} h^{p}\left(\mathrm{Z} T, e_{k}\right)+(1-\lambda)^{p} h^{p}\left(\mathbf{Z} T, e_{k}\right)$.

If $p>1$, this implies that $h\left(\mathrm{ZT}, e_{k}\right)=0$. Similarly, we obtain that $h\left(\mathrm{Z} T,-e_{k}\right)=0$. Thus ZT $=\{o\}$. So let $p=1$. Setting $f\left(x_{1}, x_{2}\right)=h^{p}(\mathrm{Z} T, x)-h^{p}\left(\mathbf{Z}^{\prime}, x\right)$ for $x=x_{1} e_{i}+x_{2} e_{j}$, we see that (37) holds. Therefore we can apply Lemma B. Since for $i \neq j, k \neq l$ there is an $\alpha \in \mathrm{SL}(n)$ such that $\alpha e_{i}=e_{k}, \alpha e_{j}=e_{l}$ and $\alpha T=T$ and since $\mathrm{Z}$ is $\mathrm{SL}(n)$ contravariant, we see that $f$ does not depend on $i, j$ and that for $x_{1}, x_{2} \in \mathbb{R}$

$$
h\left(\mathrm{ZT}, x_{1} e_{i}+x_{2} e_{j}\right)=h\left(\mathrm{ZT}, x_{1} e_{k}+x_{2} e_{l}\right) .
$$

Thus we obtain for $x_{1} \geq x_{2} \geq 0$

$$
\begin{aligned}
& h\left(\mathrm{ZT}, x_{1} e_{i}-x_{2} e_{j}\right)=x_{1}\left(f\left(e_{1}\right)+a\right)-x_{2} f\left(e_{1}\right), \\
& h\left(\mathrm{ZT},-x_{1} e_{i}+x_{2} e_{j}\right)=x_{1}\left(f\left(-e_{1}\right)+a\right)-x_{2} f\left(-e_{1}\right),
\end{aligned}
$$

and for $x_{1}, x_{2} \geq 0$

$$
\begin{aligned}
& h\left(\mathrm{ZT}, x_{1} e_{i}+x_{2} e_{j}\right)=x_{1}\left(f\left(e_{1}\right)+a\right)+x_{2} f\left(e_{2}\right), \\
& h\left(\mathrm{ZT},-x_{1} e_{i}-x_{2} e_{j}\right)=x_{1}\left(f\left(-e_{1}\right)+a\right)+x_{2} f\left(-e_{2}\right) .
\end{aligned}
$$

It follows from (38) that $f\left(e_{1}\right)+a=f\left(e_{2}\right)$ and $f\left(-e_{1}\right)-a=f\left(-e_{2}\right)$. Setting $a_{1}=f\left(e_{1}\right)+f\left(-e_{1}\right)+a, a_{2}=-f\left(-e_{1}\right), a_{3}=-f\left(e_{1}\right)$ and using (38) shows that

$$
h(\mathrm{ZT}, x)=a_{1} h(\Pi T, x)+a_{2} h\left(\Pi_{o} T, x\right)+a_{3} h\left(-\Pi_{o} T, x\right)
$$

for $x=x_{1} e_{i}+x_{2} e_{j}$. Thus by Lemma 4 the proposition is proved. 


\section{Proof of Corollaries 1.2 and 2.2}

The main tool is the following result by McMullen.

Theorem ([28]). Let $\mu: \mathcal{P}^{n} \rightarrow \mathbb{R}$ be a translation invariant valuation. Then for $s \in \mathbb{Q}, s \geq 0$,

$$
\mu(s P)=\sum_{i=0}^{n} s^{i} \mu_{i}(P) .
$$

The coefficient $\mu_{i}(P)$ (which is independent of $s$ ) is a translation invariant valuation on $\mathcal{P}^{n}$, which is homogeneous of degree $i$.

Let $\mathrm{Z}: \mathcal{P}^{n} \rightarrow \mathcal{K}^{n}$ be a translation invariant Minkowski valuation. Then for $x \in \mathbb{R}^{n}$ fixed, $P \mapsto h(Z P, x)$ is a translation invariant real valued valuation. Thus for $s \in \mathbb{Q}, s \geq 0$, there is a polynomial expansion

$$
h(\mathrm{Z}(s P), x)=\sum_{i=0}^{n} s^{i} \mu_{i}(P, x),
$$

where for every $x \in \mathbb{R}^{n}$ the coefficient $\mu_{i}(\cdot, x)$ is a translation invariant valuation on $\mathcal{P}^{n}$, which is homogeneous of degree $i$.

First, we consider Corollary 1.2, that is, $\mathrm{Z}$ is $\mathrm{SL}(n)$ equivariant. Then we have for $\phi \in \mathrm{SL}(n), x \in \mathbb{R}^{n}$,

$$
h(\mathrm{Z}(\phi P), x)=\sum_{i=0}^{n} s^{i} \mu_{i}(\phi P, x)=h\left(\mathrm{Z} P, \phi^{t} x\right)=\sum_{i=0}^{n} s^{i} \mu_{i}\left(P, \phi^{t} x\right) .
$$

Thus for $i=1, \ldots, n$,

$$
\mu_{i}(\phi P, x)=\mu_{i}\left(P, \phi^{t} x\right) \quad \text { for } \phi \in \mathrm{SL}(n), x \in \mathbb{R}^{n} .
$$

Note that a function $f: \mathbb{R}^{n} \rightarrow \mathbb{R}$ which is homogeneous of degree 1 is the support function of a convex body if and only if $f$ is sublinear, that is, $f(x+y) \leq f(x)+f(y)$ for $x, y \in \mathbb{R}^{n}$ (cf. [36]). Since $h(\mathrm{Z}(s P), \cdot)$ is sublinear for $s>0$,

$$
\mu_{n}(P, \cdot)=\lim _{s \rightarrow \infty} \frac{h(\mathrm{Z}(s P), \cdot)}{s^{n}}
$$

is also sublinear. Thus $\mu_{n}(P, \cdot)$ is a support function. In view of (40) and the homogeneity of $\mu_{n}$, we can apply Theorem 1 and obtain that $\mu_{n}(P, \cdot)=0$. Therefore

$$
h(\mathrm{Z}(s P), \cdot)=\sum_{i=0}^{n-1} s^{i} \mu_{i}(P, \cdot) .
$$

Using induction on the degree of homogeneity and the same arguments as above, we obtain that for $i=n-1, \ldots, 2, \mu_{i}(P, \cdot)$ is a support function and that by Theorem [1] $\mu_{i}(P, \cdot)=0$ for $i=n-1, \ldots, 2$. By Lemma 1 we have $\mathrm{Z}\{o\}=\{o\}$ and therefore $\mu_{0}(P, \cdot)=0$. This implies that $\mu_{1}(P, \cdot)=h(\mathrm{Z} P, \cdot)$. Thus applying Theorem 1 shows that $\mathrm{Z}=c \mathrm{D}$ with a suitable constant $c \geq 0$ and Corollary 1.2 is proved.

Now, we consider Corollary 2.2, that is, $\mathrm{Z}$ is $\mathrm{SL}(n)$ contravariant. Then we have for $i=1, \ldots, n$,

$$
\mu_{i}(\phi P, x)=\mu_{i}\left(P, \phi^{-1} x\right) \text { for } \phi \in \mathrm{SL}(n), x \in \mathbb{R}^{n} .
$$

Since $\mu_{n}(P, \cdot)=\lim _{s \rightarrow \infty} h(\mathrm{Z}(s P), \cdot) / s^{n}$ is sublinear, it is a support function. In view of (41) and the homogeneity of $\mu_{n}$, we can apply Theorem 2 and obtain that 
$\mu_{n}(P, \cdot)=0$. By Lemma 5 we have $\mathrm{Z}\{o\}=\{o\}$ and therefore $\mu_{0}(P, \cdot)=0$. This implies that

$$
h(\mathrm{Z}(s P), \cdot)=\sum_{i=1}^{n-1} s^{i} \mu_{i}(P, \cdot) .
$$

Since $\mu_{1}(P, \cdot)=\lim _{s \rightarrow 0} h(\mathrm{Z}(s P), \cdot) / s$ is sublinear, it is a support function. We apply Theorem 2 and obtain that $\mu_{1}(P, \cdot)=0$. Using induction on the degree of homogeneity and the same arguments as above, we obtain that for $i=1, \ldots, n-2$, $\mu_{i}(P, \cdot)$ is also a support function and that by Theorem $2 \mu_{i}(P, \cdot)=0$ for $i=$ $1, \ldots, n-2$. This implies that $h(\mathrm{Z} P, \cdot)=\mu_{n-1}(P, \cdot)$. Thus applying Theorem 2 shows that $\mathrm{Z}=c \Pi$ with a suitable constant $c \geq 0$ and Corollary 2.2 is proved.

\section{REFERENCES}

[1] S. Alesker, Continuous rotation invariant valuations on convex sets, Ann. of Math. (2) 149 (1999), 977-1005. MR1709308 (2000i:52019)

[2] S. Alesker, On P. McMullen's conjecture on translation invariant valuations, Adv. Math. 155 (2000), 239-263. MR 1794712 (2001k:52013)

[3] S. Alesker, Description of translation invariant valuations on convex sets with solution of $P$. McMullen's conjecture, Geom. Funct. Anal. 11 (2001), 244-272. MF 1837364 (2002e:52015)

[4] S. Campi and P. Gronchi, The L $L^{p}$-Busemann-Petty centroid inequality, Adv. Math. 167 (2002), 128-141. MR1901248 (2003e:52011)

[5] R. Gardner, Geometric tomography, Cambridge University Press, Cambridge, 1995. MR.1356221 (96j:52006)

[6] R. Gardner and A. Giannopoulos, p-cross-section bodies, Indiana Univ. Math. J. 48 (1999), 593-613. MR1722809 (2000i:52002)

[7] R. Gardner and G. Zhang, Affine inequalities and radial mean bodies, Amer. J. Math. 120 (1998), 505-528. MR 1623396 (99e:52006)

[8] E. Grinberg and G. Zhang, Convolutions, transforms, and convex bodies, Proc. London Math. Soc. (3) 78 (1999), 77-115. MR1658156 (99m:52009)

[9] H. Hadwiger, Vorlesungen über Inhalt, Oberfläche und Isoperimetrie, Springer, Berlin, 1957. MR 0102775 (21:1561)

[10] M. Kiderlen, Blaschke and Minkowski endomorphisms of convex bodies, preprint.

[11] D. Klain, A short proof of Hadwiger's characterization theorem, Mathematika 42 (1995), 329-339. MR1376731 (97e:52008)

[12] D. Klain, Star valuations and dual mixed volumes, Adv. Math. 121 (1996), 80-101. MR:1399604 (97i:52009)

[13] D. Klain, Invariant valuations on star-shaped sets, Adv. Math. 125 (1997), 95-113. MR.1427802 (98a:52009)

[14] D. Klain, Even valuations on convex bodies, Trans. Amer. Math. Soc. 352 (2000), 71-93. MR:1487620(2000c:52003)

[15] D. Klain and G. Rota, Introduction to geometric probability, Cambridge University Press, Cambridge, 1997. MR1608265 (2001f:52009)

[16] K. Leichtweiß, Affine geometry of convex bodies, Johann Ambrosius Barth, Heidelberg, 1998. MR 1630116 (2000j:52005)

[17] M. Ludwig, Projection bodies and valuations, Adv. Math. 172 (2002), 158-168. MR1942402 (2003j:52012)

[18] M. Ludwig, Ellipsoids and matrix valued valuations, Duke Math. J. 119 (2003), 159-188. MR 1991649 (2004e:52015)

[19] M. Ludwig and M. Reitzner, A characterization of affine surface area, Adv. Math. 147 (1999), 138-172. MR1725817 (2000j:52018)

[20] E. Lutwak, On some affine isoperimetric inequalities, J. Differential Geom. 23 (1986), 1-13. MR 0840399 (87k:52030)

[21] E. Lutwak, Centroid bodies and dual mixed volumes, Proc. Lond. Math. Soc. 60 (1990), 365-391. MR1031458 (90k:52024)

[22] E. Lutwak, The Brunn-Minkowski-Firey theory. I. Mixed volumes and the Minkowski problem, J. Differential Geom. 38 (1993), 131-150. MR1231704|(94g:52008) 
[23] E. Lutwak, Selected affine isoperimetric inequalities, Handbook of Convex Geometry, Vol. A (P.M. Gruber and J.M. Wills, eds.), North-Holland, Amsterdam, 1993, 151-176. MR1242979 (94h:52014)

[24] E. Lutwak, The Brunn-Minkowski-Firey theory. II: Affine and geominimal surface areas, Adv. Math. 118 (1996), 244-294. MR1378681 (97f:52014)

[25] E. Lutwak, D. Yang, and G. Zhang, $L_{p}$ affine isoperimetric inequalities, J. Differential Geom. 56 (2000), 111-132. MR1863023(2002h:52011)

[26] E. Lutwak, D. Yang, and G. Zhang, Sharp affine $L_{p}$ Sobolev inequalities, J. Differential Geom. 62 (2002), 17-38. MR1987375 (2004d:46039)

[27] E. Lutwak and G. Zhang, Blaschke-Santaló inequalities, J. Differential Geom. 47 (1997), 1-16. MR 1601426 (2000c:52011)

[28] P. McMullen, Valuations and Euler-type relations on certain classes of convex polytopes, Proc. London Math. Soc. (3) 35 (1977), 113-135. MR.0448239 (56:6548)

[29] P. McMullen, Valuations and dissections, Handbook of Convex Geometry, Vol. B (P.M. Gruber and J.M. Wills, eds.), North-Holland, Amsterdam, 1993, 933-990. MR1243000 (95f:52018)

[30] P. McMullen and R. Schneider, Valuations on convex bodies, Convexity and its applications (P.M. Gruber and J.M. Wills, eds.), Birkhäuser, 1983, 170-247. MF0731112 (85e:52001)

[31] V. D. Milman and A. Pajor, Isotropic position and inertia ellipsoids and zonoids of the unit ball of a normed n-dimensional space, Geometric aspects of functional analysis (1987-88), Lecture Notes in Math., vol. 1376, Springer, Berlin, 1989, 64-104. MR1008717 (90g:52003)

[32] C. M. Petty, Centroid surfaces, Pacific J. Math. 11 (1961), 1535-1547. MR0133733 (24:A3558)

[33] C. M. Petty, Isoperimetric problems, Proceedings of the Conference on Convexity and Combinatorial Geometry (Univ. Oklahoma, Norman, Okla., 1971), Dept. Math., Univ. Oklahoma, Norman, Okla., 1971, 26-41. MR0362057 (50:14499)

[34] C. A. Rogers and G. C. Shephard, The difference body of a convex body, Arch. Math. 8 (1957), 220-233. MR0092172(19:1073f)

[35] R. Schneider, Equivariant endomorphisms of the space of convex bodies, Trans. Amer. Math. Soc. 194 (1974), 53-78. MR0353147 (50:5633)

[36] R. Schneider, Convex bodies: the Brunn-Minkowski theory, Cambridge Univ. Press, Cambridge, 1993. MK1216521 (94d:52007)

[37] R. Schneider, Simple valuations on convex bodies, Mathematika 43 (1996), 32-39. MR:1401706 (97f:52016)

[38] G. Zhang, Restricted chord projection and affine inequalities, Geom. Dedicata 39 (1991), 213-222. MR1119653 (92f:52017)

[39] G. Zhang, The affine Sobolev inequality, J. Differential Geom. 53 (1999), 183-202. MR:1776095 (2001m:53136)

Institut für Diskrete Mathematik und Geometrie, Technische Universität Wien, Wiedner Hauptstrasse 8-10/1046, 1040 Wien, Austria

E-mail address: monika.ludwig@tuwien.ac.at 